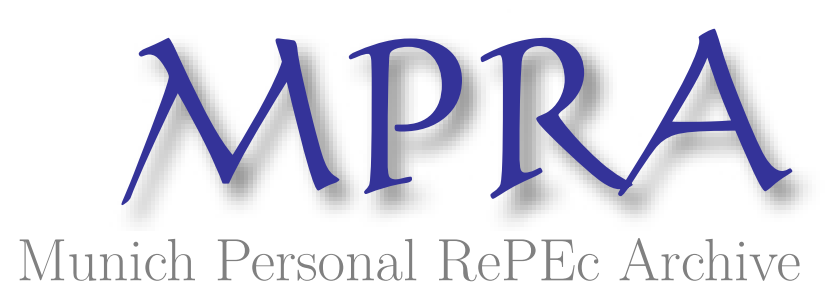

\title{
Market demand: a holistic theory and its verification
}

Gorbunov, Vladimir

Ulyanovsk State University

11 August 2021

Online at https://mpra.ub.uni-muenchen.de/109154/

MPRA Paper No. 109154, posted 23 Aug 2021 09:19 UTC 


\title{
Market demand: a holistic theory and its verification ${ }^{1}$
}

\author{
Vladimir K. Gorbunov
}

Dept. of Economics, Ulyanovsk State University, 432017 Leo Tolstoy Street, 42, Ulyanovsk, Russian Federation;

vkgorbunov@mail.ru; orcid.org/0000-0001-5276-0501

\begin{abstract}
The paper presents a revision of the contemporary reductionistic demand theory, replacing the studying object, i.e. an individual, with a fuzzy collection of market buyers, regarded as a "statistical ensemble of consumers". The new holistic market demand theory formally retains the neoclassical demand theory with reconsidering the utility function as a collective one. A nonparametric verification method is presented, which uses the economic (Konüs) and formula Fisher indexes. The method has a variational character based on the theory of ill-posed problems. Verification is carried out simultaneously with the construction of Konüs indexes. The method is demonstrated on an example of the new Giffen demand model.
\end{abstract}

Keywords Statistical consumers' ensemble · Collective utility function · Verification · Ill-posed problems $\cdot$ Konüs indexes $\cdot$ Giffen demand

JEL classification D11 - Consumer Economics: Theory; D12 - Consumer Economics: Empirical Analysis; C18 - Methodological Issues: General; C43 - Index Numbers and Aggregation; C61 - Optimization Techniques $\bullet$ Programming Models $\bullet$ Dynamic Analysis

\section{Highlights}

- Economic and scientific arguments for a holistic revision of modern demand theory

- The holistic market demand theory turns individual chaos into collective order

- Applied demand analysis freed from the problem of aggregation over consumers

- The method of variative calculating economic indexes accounting political preferences

${ }^{1}$ This paper is an elaboration of my conference paper (Gorbunov, 2021). 
"Such complicated laws as those of economy cannot be accurately traced in individual cases. Their operation can only be detected in aggregates and by the method of averages."

W.S. Jevons $(1866)^{2}$

\section{Introduction}

Contemporary neoclassical Economics refers to the Social Sciences (Humanities) with their own methodology, which is more normative than positive one, accepted in natural sciences ${ }^{3}$. It has a very developed mathematical theory of individual demand (IDTh), but does not have a theory of market demand (MDTh), which reflects reality and is of practical interest to a wide circle of practitioners, managers, politicians, and researchers. The founders of the mathematized neoclassical approach to Economics, who were Stanley Jevons (1835-1882) and Leon Walras (1834-1910), understood that the main object of the Demand Theory was not the individual, but collective market demand, and they intended to create Economics as a whole, and firstly the demand theory, on the general scientific principles like mechanics and physics. The modern notion of these principles are objectivity, provability and verifiability by facts. However, both of them confined themselves to systematisation and mathematization of the conglomerate of economic theories that existed at that time and were inconsistent mutually. They both, but independently, suggested the rationality principle in consumer behaviour that had been earlier conjectured by Hermann Gossen (1810-1858) in a more general problem of obtaining satisfaction with limited resources, and the principle was formulated by Walras as maximizing the utility of a purchasing commodity bundle under budget constraint. Significantly, that this principle was based on watching the collective market behaviour, but for some reason, contrary to Jevons' conjecture, highlighted in the epigraph above, in their seminal "Political Economies" (Jevons, 1957/1871; Walras, 1954/1877), it was imposed on an independent and rational individual consumer ${ }^{4}$. Possibly, having in mind the not unusual productivity of the reductionistic approach in the natural sciences when studying complex objects ${ }^{5}$.

Some way or other, in 1939, John Hicks, the main contributor in the demand theory of the first half of 20th century, believed (1975, p. 34), that "Market demand has almost exactly the same properties as individual demand." However, in the 1950s years, William Gorman (1953) and in a specified form Paul Samuelson (1956) stated that the conjecture about the similarity of the theories of independent individual demand and the sum of such demands was wrong ${ }^{6}$. These results only

\footnotetext{
${ }^{2}$ 18th thesis of Jevons' speech at the British Science Association in 1862 (printed in 1866).

${ }^{3}$ See more about methodological problems of Economics in M. Boumans (2021).

${ }^{4}$ Jevons, noting the abundance of statistical information about economic processes, also noted the imperfection of that time statistics: "I know not when we shall have a perfect system of statistics, but the want of it is the only insuperable obstacle in the way of making Economics an exact science." (1871, pp. 11-12).

${ }^{5}$ Firstly, in theoretical physics, reductionism is a natural method, because the main problem here is the understanding basic laws of organizing and proceeding of the Material World. Another example is the molecular-kinetic theory of the explaining macroprocesses in continuous media. But the latter are also investigated within a holistic methodology that provides possibilities to find emergent phenomena such as magnetohydrodynamics.

${ }^{6}$ According to Gorman (1953), a necessary and sufficient condition for rationalizing a market demand by a collective utility function is the parallelism of all individual Engel's lines. Samuelson (1956, p. 5) refined Gorman's result for the
} 
proved the impossibility to use the same utility maximization model of rational behaviour both for individual consumers (or buyers) and for a set of independent consumers of some market. But Samuelson concluded that this model is not applicable to a set of consumers, without questioning its applicability to individual ones. This conclusion of the most reputable neoclassical economist was unproven, but most economists have shared his position.

The doubts of a minority in the validity of the IDTh are reflected by the reputable contributors in the applied demand analysis of real markets Angus Deaton and John Muellbauer in their book (1980). Here (p. 148), in particular, the opinion of belated Hicks (1956) is expressed: "microeconomic theory has greater relevance for aggregate data." "7 But they immediately decline such doubts: "If such views are to be justified, so that the theory can be used without modification to deal with market data, clean arguments must be provided."

The well-known failure in Economics regarding the MDTh, considered more below, has led to failures in Walrasian equilibrium theory and applications that represent demand analysis in general and its beginning - the theory of consumption index numbers. The most conceivable approach to the index number problem that is the 'economic approach' developed in the frame of demand theory, refers only to households, see the international Manual (CPIM, 2004, Chs. 17, 18).

To understand the reasons for the Economics failures, it is necessary to consider its methodology. Economics is constructed within the framework of methodological individualism, the main principle of which is appropriate to highlight by the words of belated Kenneth Arrow (1994, p. 1): "the behavior we explain and the policies we propose are explicable in terms of individuals, not of other social categories." 8 And in the continuation of this quote, he objects to this Economics' touchstone: "I want to argue today that a close examination of even the most standard economic analysis shows that social categories are in fact used in economic analysis all the time and that they appear to be absolute necessities for analysis, not just figures of speech that can be eliminated if need be." Similar doubts in the impeccability of the Economics methodology have been declared, oftener over the past decades, or realized in practical researches implicitly, also by Gustav Cassel (1918), Maurice Allais (1990), Victor Polterovich (1998), Claude Hillinger (2008), Alan Kirman (2006, 2010) among others. Moreover, social categories and problems of the public economy have been studied more and more often in recent decades, see (Friedrichsen et al., 2021) and references within.

Undoubtedly, economic phenomena and processes are specific, because their elements are people with a psyche. But for a long time, many authoritative economists, especially those who came there from the natural sciences (Allais was one of them), believed that Economics, as a profession dealing with quantitative measures of prices and quantities of commodities (goods and services), should satisfy the general scientific principles mentioned above.

In papers and books (Gorbunov: 2004, 2013, 2015), mostly Russian-language, a revision of IDTh as MDTh has been elaborated with replacing the neoclassical individual by "statistical ensemble of

usual case, when the commodity space is $R_{+}^{n}$, and the Engel's lines start at the origin. Then they merge into one ray. This means equivalence and homogeneity of all individual preferences that is generally unrealistic.

${ }^{7}$ Such a position is revealed factually in the concluding sections of (Hicks, 1956).

${ }^{8}$ The term "methodological individualism" and its essence were coined by the young Joseph Schumpeter (1908), where (p. 8) he denied the social character of the concepts of "total demand" and "total supply". But later, in 1930, he co-founded the Econometric Society, which aims to transform Economics on a general scientific basis, not limited by reductionism. 
consumers" of the studied market, represented by trade statistics. At the same time, MDTh, constructed in the frame of a holistic methodology, retains the mathematical model and the results of the reductionistic IDTh with a new economic interpretation. This theory provides the theoretical basis for the holistic equilibrium theory laid down by Cassel and Abraham Wald ${ }^{9}$, the applied demand analysis laid down by Richard Stone and Angus Deaton, and calculating economic indexes for real markets.

In (Gorbunov: 2004, 2015), methods for verifying MDTh have been created in the framework of nonparametric analysis (Afriat, 1967; Varian: 1982, 1983) and the theory of ill-posed problems (Tikhonov and Arsenin, 1977). The key problem here is solving the Afriat's inequalities, which determine utility values and Lagrange multipliers for statistical commodity quantities and expenditures. These inequalities may be unstable, and methods for solving them are based on special regularization methods (Gorbunov: 1991, 1999, 2001a, 2001b). Recent papers (Gorbunov and Lvov, 2019; Gorbunov et al., 2020) present and approve variative verification methods for choosing solutions to regularized Afriat's inequalities with different desired properties. These properties are expressed through economic (Konüs / analytical) indexes.

The rest of the paper is organized as follows. Section 2 briefly reviews the problems of the microeconomic demand theory. Section 3 provides meaningful general scientific and economic 'clean arguments' for the revision of the IDTh as the MDTh with the same mathematical model. Section 4 presents the variative nonparametric verification method with simultaneous constructing Konüs indexes. In Section 5 the Giffen demand model, its analysis and verification by the suggested method are presented. Section 6 concludes the presentation.

\section{A short survey of the problems of demand theory and its applications}

This section examines the current state of neoclassical demand theory and the implications of the absence of a market demand theory for equilibrium theory, the economic approach to index number constructing, and applied demand analysis. Some attempts to overcome these failures are discussed.

\subsection{The current state of the IDTh}

The current state of the neoclassical IDTh is comprehensively, without avoiding failures, presented in the textbook (Mas-Colell et al. 1995, Chs 1-4), and shorter, but strictly and newly in (Jehle and Reny, 2011). The first book describes two approaches to mathematical modelling of an individual consumer's rational behaviour regarding the choice from the attainable set of commodities: preference-based and choice-based. Until now, only the preference-based approach has been used for solving real demand analysis problems ${ }^{10}$. Here, a primitive characteristic of an individual in an abstract style, introduced by Gérard Debreu (1959), is a binary Preference Relation (PR) defined in a commodity space, and PR is assumed to be rational, i.e., it is complete and transitive. Analytical demand theory is constructed under the additional assumption of the PR continuity, providing its representation through a continuous utility function. The rationality principle in the analytical form

\footnotetext{
${ }^{9}$ See (Gorbunov: 2013, 2018), where the first paper presents a modified Walras-Cassel equilibrium model, but with generalized market demand, and the second presents developing the Walras-Cassel-Leontief model (Morishima, 1964).

10 The analytical choice-based demand theory is developed and used in equilibrium models in my papers, see (Gorbunov 2013, 2018) and references therein.
} 
is the classical utility maximization principle of Jevons and Walras. Under the assumption of demand regularity that means hereinafter the single-valuedness and differentiability of the demand correspondence, the classical IDTh has a deeply developed meaningful mathematical theory. The profound results of this theory were obtained in the seminal papers of Evgeny Slutsky (1915), John Hicks with Roy Allen (1934) ${ }^{11}$, and in the book of Hicks (1975/1939).

\subsection{The failures of the IDTh}

Simplifying the formalization of the studied object in science is inevitable, and to find effective simplification that provides nontrivial development of positive scientific knowledge about an object is a matter of art and intuition.

\subsubsection{Equilibrium theory}

Arrow and Debreu (1954) modified the Walras' equilibrium model on the fully reductionistic base with two sets of agents: firms-producers and consumers, each of them acted independently and rationally in accordance with their individual goals and possibilities. The authors proved the existence of equilibrium prices, and this paper became the seminal one for the mainstream direction of the contemporary Economics; but in the frame of this model, it appeared impossible to prove the uniqueness of the equilibrium except of some unrealistic assumptions concerning individual preferences. Moreover, in this model the typicality of pathologies of the equilibrium sets was revealed (Mas-Colell et al., 1995, Section 17.E, Sonnenschein-Mantel-Debreu theorem). These pathologies attract mathematicians interested in economics, see (Balasko, 2016) and references therein, but the equilibrium theory, which is more adequate to reality and may be of practical interest, must have a unique equilibrium.

So, the 'impossibility results' of Gorman-Samuelson revealed that the reductionistic approach for creating the market demand theory turned out to be ineffective for modelling the economic system. This is the fundamental failure of Economics for resolving the main economic theoretical and practical question, which is the Value / Price Theory in positive setting. More about these failures are written in the cited above works of Hillinger, Kirman, Polterovich, and other works.

\subsubsection{Applied demand analysis}

The most famous pioneer in this problem R. Stone (1945) researched the United Kingdom and the USA markets over the periods 1920-38 and 1929-41 correspondingly with a very limited aim of the "approximation to the market demand functions for individual goods and services". He used simple regression analysis without explicit accounting the interrelation between consumption different commodities. Such a simple approach is explained by the absence of calculation methods and technical means for complex computational problems in the early 1940s. Soon after the War, such means appeared, and Stone posed and successfully solved the problem of a comprehensive analysis of market demand based on the theory of individual demand. In his paper (1954) he laid a method for creating market demand functions in some parametric class without using utility functions, but with properties corresponding to maximizing utility, namely: homogeneity of the zero-

${ }^{11}$ Samuelson (1974) has characterized this Reconsideration as the "revolution in demand theory." 
degree, expenditure identity (Walras' Law), negative semi-definiteness and symmetry of the Slutsky matrix, which are so-called integrability conditions. He has used the Linear Expenditure System (LES), satisfying these properties, that was suggested by L. Klein and H. Rubin (1948) as individual demand functions with the goal "to express the index of the cost of living in terms of measurable phenomena which are independent of the subjective concepts of utility." Soon, R. Geary (1951) found a utility function that rationalized the LES.

Stone's work on market demand analysis was fulfilled in a naive period of believing that the utility maximizing model accepted for the IDTh could also be appropriated for a theory of market demand, in which demand was understood as the sum of independent individual ones. The development of his approach to the demand analysis was carried out after Gorman and Samuelson revealed the practical incompatibility of the IDTh and the MDTh constructed identically. The main contributor to applied demand analysis since this period was Deaton. Considering Gorman result, in addition to the rationalizability conditions for the demand system, the problem emerged to find conditions providing the aggregation over consumers within the identical IDTh and MDTh (Deaton, 1975). It turned out that LES satisfied such conditions in the Gorman variant, but constructing more flexible nonlinear demand systems has become more difficult. Such systems (Rotterdam model, Translog functions, Almost Ideal Demand System, etc.) and statistical methods, both parametric and nonparametric, were constructed in the works of H. Theil, W. Barnet, D. Jorgenson, A. Deaton and others; see (Deaton and Muellbauer: 1980a, 1980b), surveys (Deaton, 1986; Barnett and Serletis, 2008), a recent paper (Boysen, 2019) and references therein. The problem of aggregation over consumers very complicates ${ }^{12}$ and restricts the market demand analysis, but ignoring this problem makes this analysis heuristic within the framework of modern Economics, in which individual rationality and independence are accepted without questioning.

\subsubsection{Index Numbers}

The initial and important part of the market demand analysis is the theory and practice of consumption index numbers, and this is a social category. In statistical practice, several of the large set of price and quantity indexes represented by algebraic formulas (such as Laspeyres, Paasche, etc.) are used, and they give different results (CPIM, 2004, Chap 19). To obtain objectivity when comparing different index formulas, Irving Fisher suggested a system of tests (axioms) that should be performed for 'correct indexes.' The best pair of price and quantity indexes turned out to be, on his mind, the pair of Fisher 'ideal indexes,' which are the geometric mean values from the Laspeyres and Paasche ones. Nevertheless, the Fisher pair of indexes does not obey the transitivity test, and "there is no universal agreement on what the 'best' set of 'reasonable' axioms is." (Ibid., 2004, par. 16.2, p. 289). This allows to choose indexes depending on the goals of economic analysis or various groups influencing economic policy. Thus, the government is interested in optimistic assessments of the socio-economic situation, while trade unions and the opposition are interested in pessimistic ones. Relativism of modern indexology is reflected in W. Erwin Diewert (2001).

To diminish subjectivity in the index problem, the 'economic approach' to constructing demand indexes was created within the demand theory through the expenditure function. The pioneer of this

${ }^{12}$ See the survey of Deaton's works by Belyanova and Nikolaenko (2016). 
approach was the soviet mathematical economist Alexander Konüs (1895-1990). The founder (among others) and the first president of the Econometric Society, Ragnar Frisch (1936), got acquainted with the Russian paper of Konüs (1924) before its English translation was issued in 'Econometrica' (1939), and he paid great attention to the Konüs Cost of Living Index and methods for its construction. The idea to construct Index Numbers within the demand theory was developed in a number of Western works, of which the paper of P. Samuelson and S. Swamy (1974) is seminal ${ }^{13}$. But due to the lack of an adequate MDTh in worldwide literature, these economic / Konüs indexes are intended in CPIM and in the literature of other authors only for individuals or households.

\subsection{The known alternative demand theories}

\subsubsection{The first holistic experience}

Cassel in his tractate (1918/1967) has rejected the individualistic demand theory of Walras and factually suggested the holistic approach ${ }^{14}$ to the theories of demand and equilibrium. Considering the Walrasian equilibrium model, Cassel retained its multisectoral production system and described the market demand directly as a whole object. In the early 1930s, Wald (1951) has investigated the modified Walras-Cassel equilibrium model and introduced an assumption on market demand, which coincided with the Weak Axiom (WA) suggested by Samuelson later. With this 'Wald Condition', he has proved the existence and uniqueness of equilibrium, but this finding was unjustifiably disqualified in Economics (Arrow and Debreu, 1954; Dorfman et al., 1958, Ch. 13, p. 368). Sometimes the Walras-Cassel equilibrium model is being used as a formal example of an economy with a unique equilibrium (Morishima, 1964).

\subsubsection{Hildenbrand's market demand}

Hildenbrand's Market Demand theory was stimulated by his dissatisfaction with the Economics' failure regarding equilibrium theory laid down by Arrow and Debreu (1954); and his book's (1994) aim is to construct " $a$ theory of 'market demand' with the aim of establishing some 'useful' properties of the market demand function." (pp. ix-x). The author does not deny the IDTh, and his attempt to overcome the failures of neoclassical Economics leaves this theory as the basis of the new MDTh of positive kind. But at the same time, he expresses accordance with the view of belated Hicks, that WA, which is one of the rationality principles of the neoclassical IDTh, "is better justified for aggregate behavior, that is to say, for mean demand, than for individual behavior." (ibid., p. 119).

Briefly and formally, the Hildenbrand's MDTh is as follows. An element of a market of $l$ commodities that forms market demand is not an individual whose behaviour is more spontaneous than rational and is not reflected in statistics, but a household; and the market "refers to a large group of households called the 'population' of households ... considered at a certain 'time period'." (ibid., p. 30). Denote a commodity space as $R_{+}^{l}=\left\{q: q_{i} \geq 0, i=\overline{1, l}\right\}$, and the corresponding (conjugate) price space as $R_{+}^{l^{*}}=\left\{p: p_{i} \geq 0, i=\overline{1, l}\right\}$. The household demand functions have specification $f^{h}\left(p, x^{h}\right)$ , where $h$ is a household number, and $x^{h}-$ its income. The market demand function $F(\cdot)$ is a one-

\footnotetext{
${ }^{13}$ See also a survey of Diewert (1993) and his chapters 17 and 18 from CPIM (2004) on the 'economic indexes'.

14 The notions of holism and reductionism entered into the scientific methodology later.
} 
valued correspondence from $R_{+}^{l^{*}}$ to $R_{+}^{l}$ and is defined as some averaging of the functions $f^{h}$ over a given distribution in the space of household characteristics $\left(x^{h}, f^{h}\right)$, which are elements of the Cartesian product $R_{+} \times \mathcal{F}$, where $\mathcal{F}$ is the space of admissible demand functions. The main 'useful' properties of the correspondence $F(\cdot)$, which is created by the aggregation procedure, is the strictly decreasing, i.e., the negativity of the inner product $\left\langle p^{\prime}-p^{\prime \prime}, F\left(p^{\prime}\right)-F\left(p^{\prime \prime}\right)\right\rangle$ for every different $p^{\prime}, p^{\prime \prime} \in R_{+}^{n^{*}}$. Meaningfully, this mathematical property is the generalized Cournot Law of Demand. The author has investigated the equilibrium problem and presented some equilibrium properties similar to those provided by WA.

The dependence of sales only on prices assumes that the level of total expenditure is fixed, and with that, this MDTh becomes strictly static. It is sufficient for a static equilibrium theory, but the Slutsky-Hicks market demand analysis, which is the cornerstone of Stone-Deaton demand analysis, is excluded. Besides, the Hildenbrand's MDTh does not resolve the problem of economic indexes because here the expenditure function cannot be defined. The availability of cross-section data about household expenditures in many countries provides the possibility to verify the Hildenbrand's MDTh; but in general, this theory cannot be verified on the base of standard trade statistics, which is a time series of the market price-quantity pairs because total expenditures usually differ with time.

\subsubsection{Small group models}

Chapter 18 of CPIM (2004) (written by Diewert) describes an attempt to extend the economic indexes for an economy with household groups. The variants of the group Konüs indexes (plutocratic, democratic) presented here are based on the sum of nonobservable individual characteristics of households. Many works develop models of collective consumer behaviour of small groups and households, detailing interactions between their members. For example, the papers of Chiappori and Ekeland (2009) and of Cherchye et al. (2010) ${ }^{15}$ develop this attractive but rather complex approach. It seems obvious that applying these models of group behaviour to the problem of market demand demands more detailed non-standard statistical data for verifying the models.

The prominent followers of the neoclassical mainstream R. Harstad and R. Selten (2013, p. 496) expressed confidence that "the collection of alternative models has made little headway supplanting the dominant paradigm. We delineate key ways in which neoclassical microeconomics holds continuing and compelling advantages over bounded-rationality models, and suggest, via a few examples, the sorts of further, difficult pushes that would be needed to redress this state of affairs."

\section{Market demand: utility maximizing theory}

To this section, which presents general scientific and economic arguments for the revision of the neoclassical demand theory, the words of Frisch (1929, p. 391) are very relevant: "The raison d'etre of the following observations lies not in the originality of the formulae but in their economic interpretation."

${ }^{15}$ See more in my review Zbl 1248.91034 on https://zbmath.org. Accessed 26/05/21. 


\subsection{Market complexity denies reductionism}

As mentioned in the Introduction, the reductionistic approach in the natural sciences and engineering often demonstrates its productivity when studying complex objects. Now, after tremendous achievements in the sciences and engineering, it is clear that the fundamental factors of this productivity are the following. Elements of physical systems, which are molecules, atoms, and their particles, are identical in their classes. Homogeneous continuous media and manufactured machines of one type can also be considered identical. The possibility of repeating experiments under similar conditions makes it possible to investigate the inevitable measurement errors by probabilistic methods. These features for such objects have simplified the formalization of their research and the application of mathematical and statistical methods to create and verify many physical laws and predictive theories, as well as complex technical systems.

But reductionism has limits as a research methodology in natural science and engineering, where it was revealed rather long ago that complex phenomena are not completely explained by the properties and mechanical movement of their elements. Quite often, in physical, biological, and other systems, with growing their complexity, new phenomena emerge that their parts do not have on their own. In cybernetics, such a systemic property is called 'emergence'. An eminent American physicist Philip Anderson (Nobel laureate of 1977), in his famous paper of (1972) "More is different...", analysing the excessive enthusiasm for reductionist methodology in physics and other sciences, has claimed that "The reductionist hypothesis ... is accepted without question." (p.393). He very convincingly showed on an example of condensed matter physics, that 'more' (regarding complexity) often leads to the emergence of new phenomena, i.e., Andersen has demonstrated the emergence property as an argument against unbounded reductionism in physics. In addition, he has considered two lists of sciences located in the hierarchy, $X$ and $Y$, such that "the elementary entities of science $X$ obey the laws of science $Y$ "; and he noted that "this hierarchy does not imply that science $X$ is " $j u$ st applied Y»." According to the Anderson's lists and the last remark, social sciences are not applied psychology. In conclusion, he recalled the dialectical principle of Karl Marx: "quantitative differences become qualitative ones."

The market system as a part of the economy is much more complicated than the systems studied in physics. Market behaviour is inherently collective; an individual does not have complete information about commodities and prices on the market, and people are subject to mutual and external influences: traditions, fashion, mood, advertising. The assumption of individual rationality is being criticized for decades in the research literature (but never in the education one) beginning from the Herbert Simon's paper (1972), where the notion of bounded rationality was introduced and deeply discussed.

Thus, the long experience of the failures of the modern neoclassical demand theory makes a natural conclusion that the reductionist approach to the problem of market demand turned out oversimplified to build an adequate scientific theory of such a complex object.

\subsection{Statistical ensemble of consumers and collective rationality}

Certainly, collective demand is the sum of individual demands in the market, but the set of buyers on almost any market is not observed and is changeable. People spend their money in different markets, and the real market process can seem chaotic. But when the market is not subject to strong 
shocks, trade statistics usually shows steady dynamics that makes it possible revealing objective patterns in the collective behaviour of the market consumers.

Unlike the neoclassical IDTh, in the presenting MDTh no assumptions that people are rational and independent agents of the economy, knowing all about market prices and commodities. To replace this unrealistic object of research with a real one, it is relevant to define the changeable population of market buyers in terms of the fuzzy sets' theory by Lotfi Zadeh (1965). The notion of 'fuzzy set' reflects a situation when some elements of a given 'universal set' $U$ can be thought of elements, belonging to some subset $C \subseteq U$, possibly, not completely, but in some grade; like a random event can take place with some probability.

Definition 1. The subset $C$ of the universal set $U$ is called the 'fuzzy subset' of $U$, if the belonging of $u \in U$ to $\mathrm{C}$ is characterized by a 'membership function' $\mu_{C}: U \rightarrow[0,1]$ with the value $\mu_{C}(u)$ representing the "grade of membership" of $\mathrm{u}$ in $\mathrm{C}$.

So, if $\mu_{C}(u)=m$ and $m=0$, then $u$ does not belong to $C$, if $0<m<1$, then $u$ belong to $C$ partially, in grade $m$, and if $m=1$, then $u$ belong to $C$ completely. Mathematically fuzzy subset $C$ of $U$ is the support of its membership function $\mu_{C}$.

Based on this Zadeh concept, the next definition of a fuzzy set of the market's consumers was given (Gorbunov, 2013), taking the universal set $U$ as the population of a region / country / world.

Definition 2. The "Statistical Ensemble of Consumers" (SEC) of the studied market is a fuzzy subset $C$ of the set of all potential consumers $U$, the membership function $\mu_{C}$ of which represents for each $u \in U$ the share of the consumer's expenses in this market from all his expenses.

The notion SEC is only conceptual and not observable for real markets, as are individual utility functions of IDTh. There is no need to reveal fuzzy characteristics of the SEC, because market demand, which is the main object of the demand theory and is generated by the SEC, is the observable object. In the presenting MDTh market demand is thought in a specification similar to that of microeconomics, with the main factors 'prices - total expenditure of all consumers' that are observable $^{16}$. This holistic alternative to neoclassical IDTh remains static, and the following is assumed:

Assumption 1. There is statistical stability in the studied market regarding the dependence of the commodity quantities' sales on their prices and the total expenditure of all consumers in the market.

The dependence in Assumption 1 is the market demand. Rejecting the assumption of individual rationality, it is necessary to explain assumptions about collective rationality. A more realistic assumption respectively individual market awareness is that the whole consumer community knows all about the market! ${ }^{17}$ Accordingly, the next assumption about the rationality of averaged collective preferences will be more intuitively grounding than for individual ones.

\footnotetext{
${ }^{16}$ In microeconomics, the second factor of market demand is 'aggregate wealth' (Mas-Colell et al., 1995, Ch. 4), which is not observable.

17 This assumption corresponds to the conclusion of the aforementioned Arrow's paper (1994, p. 9), in which he argued about the essentiality of such "social variable" as Knowledge in studying the economy.
} 
Assumption 2. Most of individuals only want to be rational, these wants determine the dominant behaviour of market's consumers, and SEC is the bearer of a collective preference relation (CPR) that can be recovered using trade statistics.

These assumptions have the status of hypotheses, and they are sufficient to construct the classical variant of MDTh, considering the given market as a black box, represented by trade statistics of prices and quantities of commodities sold over several periods.

\subsection{The general collective utility maximization problem}

This subsection represents the general regular case when a CPR can be represented analytically through a quasi-concave utility function, and the next subsection refinements the MDTh when the representing utility function can be strictly concave.

Consider a market of $n$ final commodities. Denote the commodity space $R_{+}^{n}=\left\{x \in R^{n}: x_{i} \geq 0, i=\overline{1, n}\right\}$, which vectors are columns, and they represent total bundles of commodity bought in the market during some period. The rational and continuous $\mathrm{CPR} \succeq$, is defined on some consumption set $X \subseteq R_{+}^{n}$, and its analytical representation is a continuous function $u: X \rightarrow R_{+}$, i.e., $x \succeq y \Leftrightarrow u(x) \geq u(y)$, where $x$ and $y$ belong to $X$. Such a function $u(\cdot)$ is the ordinary collective utility function. Hereinafter, the attributes 'ordinary' and 'collective' are omitted and the additional properties of monotone increasing and convexity are imposed on CPR, and with these properties the representing utility function can be monotone increasing and quasiconcavity.

It is assumed that the choice of consumers is determined by the prices of commodities $p$, which are also vectors of $R_{+}^{n}$, and the total expenditure of all buyers $e=p_{1} x_{1}+\ldots+p_{n} x_{n} \triangleq\langle p, x\rangle^{18}$.

Within the holistic concept, the model of collective consumer choice in the market is introduced, which formally coincides with the neoclassical Jevons-Walras' model of individual choice, but applied to the SEC and has the status of a hypothesis. The bought bundle $x \in R_{+}^{n}$ is assumed to maximize the collective utility $u(\cdot)$ with the expenditure constraint:

$$
D(p, e)=\operatorname{Arg} \max \{u(x):\langle p, x\rangle=e, x \geq 0\} .
$$

Below, the indirect utility function $v(p, e)=u(x(p, e)), x(p, e) \in D(p, e)$, is also used. When constructing a nontrivial and productive demand theory, all prices are assumed to be positive, i.e., $p \in R_{++}^{n}$. The extremal market demand correspondence $D(\cdot, \cdot): R_{++}^{n+1} \rightarrow 2^{R_{+}^{n}}$ represents the market's collective rationality. Due to the compactness of the admissible set and the continuity of the objective function, this problem is solvable for any $(p, e) \in R_{++}^{n+1}$, and the correspondence $D(\cdot, \cdot)$ is compact.

Thus, all demand properties derived from the utility maximization (1) are true for the MDTh. According to the Jevons believing (1866),

- $\quad$ problem (1) in the holistic setting turns individual chaos into collective order.

\footnotetext{
${ }^{18}$ In analytical context, price vectors are elements of the dual space of linear functionals $R^{n *}$. In linear algebra, the inner product is defined for vectors of the same Euclidean space $E^{n}$, which are columns, and $e=p^{T} x$.
} 
The rest of this subsection presents the three basic characteristics of market demand deduced from model (1) in its regular variant, important for verifying the MDTh and its applications.

The first two characteristics relate to the general demand correspondence (1):

1) homogeneity of degree zero: $D(t p, t e)=D(p, e), \quad t>0$;

2) expenditure identity (Walras' Law): $\langle p, x(p, e)\rangle=e$ for $\forall x(p, e) \in D(p, e)$.

The third basic characteristic is determined within the meaningful analytical theory of market demand, deduced from the regular variant of model $(1)$, where the correspondence $D(\cdot, \cdot)$ is a singlevalued and continuously differentiable Walrasian (ordinary) market demand function $x(\cdot, \cdot): R_{++}^{n+1} \rightarrow R_{+}$with values

$$
x(p, e)=\arg \max \{u(x):\langle p, x\rangle=e, x \geq 0\} .
$$

Regularity conditions in terms of CPR are rather complex ${ }^{19}$, but they can be implicitly imposed on the utility function, assuming its twice continuous differentiability and strict quasiconcavity, as is customary in the economic literature. These conditions ensure the single-valuedness and continuous differentiability of the correspondence $D(\cdot, \cdot)$, which becomes a regular demand function $x(\cdot, \cdot)$.

To avoid technical difficulties that are superfluous for economic theory, model (2) is usually considered in the field of positive (nondegenerate) solutions $x(p, e)>0$ that are of practical interest. In this regular positive case, problem (2) is being solved and analysed by the classical method of Lagrange multipliers. The characteristic system of the Lagrange method, which defines the demand values $x(p, e)$ and the multiplier $\lambda(p, e)$, is the system

$$
\frac{\partial u_{i}(x)}{\partial x}-\lambda p_{i}=0, i=\overline{1, n} ;\langle p, x\rangle-e=0
$$

The multiplier $\lambda(p, e)$ plays an important role in the theory of economic indexes and in the MDTh verification method presented further.

The second rationality principle in demand theory is the minimization of consumers expenditure, ensuring a given level of consumption $w$, which is the dual (or reciprocity) problem for (2):

$$
e(p, w)=\min \{\langle p, x\rangle: u(x)=w, x \geq 0\} .
$$

The value $e(p, w)$ of problem (4) is called the expenditure function $e(\cdot, \cdot): R_{++}^{n+1} \rightarrow R_{++}$. The solution of (4) in the regular case is the single point $h(p, w) \in R_{+}^{n}$, which is the value of the Hicksian compensated demand function $h(\cdot, \cdot)$, and the equality $e(p, w)=\langle p, h(p, w)\rangle$ holds.

The extremal problems (2) and (4) is interrelated by the duality relations between their values and demand functions: $e(p, v(p, e))=e, v(p, e(p, w))=w ; \quad x(p, e)=h(p, v(p, e)), \quad h(p, w)=$ $x(p, e(p, w))$ (Mas-Colell et al., 1995, Sec. 3.E). The first pair of these relations means that the

${ }^{19}$ See, for example, Mas-Colell (1985). 
functions $v(p, \cdot)$ and $e(p, \cdot)$ are reciprocally inverse. The significance of regular problem (4) consists in the following facts:

a) the expenditure function $e(\cdot, \cdot)$ defines the economic indexes (presented below);

b) the Hicksian demand satisfies (ibid., Prop. 3.E.4) the Hicksian Compensated Law of Demand

$$
\left\langle p-p^{\prime}, h(p, w)-h\left(p^{\prime}, w\right)\right\rangle \leq 0, \forall p, p^{\prime} \gg 0 ;
$$

c) the Hicksian substitution matrix ${ }^{20}$

$$
H(p, w) \triangleq \frac{\partial h(p, w)}{\partial p}=\left\{\frac{\partial h_{i}(p, w)}{\partial p_{j}}, \quad i, j=\overline{1, n}\right\}
$$

is negative semidefinite, symmetric, and the equality $H(p, w) p=0$ holds (ibid., Prop. 3.G.2).

The negative semidefiniteness of matrix (5) implies that $\partial h_{i}(p, w) / \partial p_{i} \leq 0$ for all $i$. Respectively, two items of commodities, $i$ and $j$, with $\partial h_{j}(p, w) / \partial p_{i} \geq 0$ are considered as substitutes, and with $\partial h_{j}(p, w) / \partial p_{i} \leq 0$ they are complements (Mas-Colell et al., 1995, p.70) ${ }^{21}$.

Revealing the properties of substitutability and complementarity on multicommodity markets is very important, but the compensated demand, defining the Hicksian substitution matrix (5), is unobserved. E. Slutsky (1915) obtained the representation of matrix (5) through the observed ordinal demand $x(p, e)$ and its derivatives (Mas-Colell et al. 1995, Prop. 3.G.3):

$$
\frac{\partial h_{i}(p, v(p, e))}{\partial p_{j}}=\frac{\partial x_{i}(p, e)}{\partial p_{j}}+\frac{\partial x_{i}(p, e)}{\partial e} x_{j}(p, e), \quad i, j=\overline{1, n}
$$

This representation is called the Slutsky Equation. The right-hand side of (6) represents the entries

$$
s_{i j}(p, e) \triangleq \frac{\partial x_{i}(p, e)}{\partial p_{j}}+\frac{\partial x_{i}(p, e)}{\partial e} x_{j}(p, e)
$$

of the Slutsky substitution matrix

$$
S(p, e) \triangleq \frac{\partial x(p, e)}{\partial p}+\frac{\partial x(p, e)}{\partial e} x^{T}(p, e)
$$

Due to (6) $S(p, e)=H(p, v(p, e))$, and the matrix (7) has the same mathematical and economic properties as the Hicksian substitution matrix $H(p, w)$ : negative semidefiniteness, symmetry, and $S(p, e) p=0$. Accordingly, the diagonal entries $s_{i i}(p, e) \leq 0$, and the characteristics of substitution

\footnotetext{
${ }^{20}$ Below the matrix notations are used for the derivatives of the vector functions $h(p, w)$ and $x(p, e)$. Herewith, $\partial h_{i}(p, w) / \partial p_{j}$ and $\partial x_{i}(p, e) / \partial p_{j}$ are the coefficients of the $i$-th row and $j$-th column of corresponding matrixes.

${ }^{21}$ The verbal Hicksian definition of substitutes and complements (Hicks, 1939, Ch. 3) corresponds to the strict inequalities.
} 
and complementarity of pairs of commodities $i$ and $j$ are determined by the sign-definiteness of the entries $s_{j i}(p, e) \geq 0$ and $s_{j i}(p, e) \leq 0$, respectively.

The properties of negative semidefiniteness and symmetry for the Slutsky matrix (7) together represent the third basic characteristic of the Walrasian market demand function (2). The significance of the presented three basic characteristics of the demand function, namely, homogeneity of degree zero, expenditure identity, negative semidefiniteness and symmetry of its Slutsky matrix, is that they, derived as necessary conditions for a regular demand function, are also sufficient conditions for a continuously differentiable function $x(p, e)$ to be a demand function rationalized by a utility function that is strictly increasing, strictly quasiconcave, and twice continuously differentiable. The latter means that these three characteristics of the demand function resolve the integrability problem of demand theory (ibid., 3.H) ${ }^{22}$.

The integrability characteristics and the expenditure function (4) have a keynote significance for verification of the MDTh and constructing economic indexes.

\subsection{Particularity with a strictly concave utility function}

Many, but not all, known strictly quasi-concave utility functions, can be transformed by a continuous increasing function into equivalent strictly concave ones, i.e., they can be strictly concave transformable / concavified. The problem of concavifiability is thorough investigated in the book by M. Avriel et al. (1988, Ch. 8). However, as will be explained in paragraph 4.2.1, the second case has the 'general position' for the real demand analysis, and there is no need to delve into the problem of concavifiability of strictly quasi-concave utility functions.

In the case of regular model (2) with a strictly concave utility function, the Slutsky matrix is being refined. This variant, when the utility function is twice continuously differentiable, and its Hessian

$$
U(x)=\frac{\partial^{2} u(x)}{\partial x^{2}}
$$

is negative definite, is considered in the book of M. Intriligator (1971, Ch. 7). There, the algebraic representation of the Slutsky matrix (7) was obtained (p. 158, (7.4.21)) by the variation method, applied to system (3), and using expenditure compensation for price changes according to Slutsky (1915):

$$
S(p, e)=\lambda(p, e) U^{-1}(x(p, e))\left[I_{n}-\frac{p p^{\mathrm{T}} U^{-1}(x(p, e))}{p^{T} U^{-1}(x(p, e)) p}\right] .
$$

This matrix, as a particular case of $(7)^{23}$, is also negative semidefinite, symmetric, and $S(p, e) p=0$. Intriligator also writes that "From negative semidefiniteness it follows that all own

\footnotetext{
${ }^{22}$ Now it is known that the homogeneity of the degree zero of demand $x(p, e)$ is a consequence of the expenditure identity and the Slutsky matrix symmetry (Jehle and Reny 2011, Theorem 2.5). Accordingly, the sufficient integrability characteristics of a continuously differentiable function $x(p, e)$ are expenditure identity, negative semidefiniteness and symmetry of its Slutsky matrix (ibid., Theorem 2.6).

${ }^{23}$ Matrix (8) can be deduced from (7) by determining the ordinal demand's variations from system (3).
} 
substitution effects are negative:" $s_{i i}(p, e)<0$ (p. 159, (7.4.26)). The negativity of the diagonal entries in (7) is really true, but additional arguments are needed to prove this. Here is a corresponding theorem based on (Ashmanov 1984, pp. 125-126).

Theorem 1. Let the regular demand $x(p, e)$ be rationalizable by a strictly increasing, strictly concave, and twice continuously differentiable utility function $u(x)$. Then the null-space of its Slutsky matrix, which has the form (8), with $p>0$ is one-dimensional, determined by the vector $p$, and the diagonal entries of the matrix are negative: $s_{i i}(p, e)<0$.

Proof. The inequality $z^{T} S z \leq 0$ for all $z \in R^{n}$ and the equality $S(p, e) p=0$ hold for matrix (8), which is a particular case of matrix (7).

To prove the one-dimensionality of the null-space of matrix (8), consider a vector $z \neq \alpha p$ for any number $\alpha$. Such a vector can be represented as $z=\alpha p+r$ with a nontrivial vector $r$, so that

$$
r^{T} U^{-1} p=0
$$

Really, substituting $r=z-\alpha p$ into (*), we have $z^{T} U^{-1} p=\alpha p^{T} U^{-1} p$, i.e. the equality (*) holds for

$$
\alpha=\frac{z^{T} U^{-1} p}{p^{T} U^{-1} p} \text {. }
$$

Using $S p=p^{T} S=0,(8),(*)$, and negative definiteness of $U^{-1}$, we receive

$$
\left(\alpha p^{T}+r^{T}\right) S(\alpha p+r)=r^{T} S r \stackrel{(8)}{=} \lambda\left[r^{T} U^{-1} r-\frac{\left(r^{T} U^{-1} p\right) p^{T} U^{-1} r}{p^{T} U^{-1} p} \stackrel{(*)}{=} \lambda r^{T} U^{-1} r<0 .\right.
$$

Thus, the quadratic form $z^{T} S z \leq 0$ for any $z$, and it equals zero only if $z=\alpha p$ with any $\alpha$, as required for proving the one-dimensionality of the matrix (8) null-space.

Consider the value of the quadratic form $z^{T} S(p, e) z$ with the basis vector $z=e^{i}$, i.e., $e_{i}^{i}=1$, and $e_{k}^{i}=0$ if $i \neq k$. Since $p>0$, then $e^{i} \neq \alpha p$ for any $\alpha>0$, therefore, $s_{i i}(p, e)=$ $\left(e^{i}\right)^{T} S(p, e) e^{i}<0$.

In view of negativity $s_{i i}(p, e)<0$, the characteristics of commodities $i$ and $j$ to be mutual substitutes or complements can be determined by the sign of the entries of (7) as $s_{j i}(p, e)>0$ and $s_{j i}(p, e)<0$, respectively. This definition, as noted above, corresponds to Hicks (1939).

It is known (Afriat's theorem, presented below) that in the real case, if a utility function adequate to the studying market can be constructed on a finite set of trade statistics in the class of locally nonsatiated functions, then such a function can be constructed in a more transparent class of increasing concave functions. It means that the second case has the 'general position' for the real demand analysis. 


\section{Verification of the MDTH}

The verification of the MDTh deduced from the behavioural model (1) is to clarify the question: is there a utility function $u(\cdot)$ that rationalizes the demand correspondence $D(\cdot, \cdot)$ when only a finite set of statistical data of "prices - quantities" pairs

$$
\left\{p^{t}, x^{t}: t=\overline{0, T}\right\}
$$

is known? These data also determine total expenditures of all buyers over the $t$-period $e_{t}=\left\langle p^{t}, x^{t}\right\rangle$. The answer to the question can be only in principle, and it can be constructive. The latter means that either a utility function has constructed that rationalizes data (9), or a demand function that satisfies the integrability conditions and satisfactorily approximates the data (9).

In a real case, the dataset (9) is only an approximate image of a set of ideal values formed in complex processes of collecting, averaging, and aggregating prices and quantities of elementary commodities. There are usually no probabilistic characteristics of trade statistics' errors. Therefore, standard econometric methods for verifying MDTh are inapplicable, and here a method for verifying the theory of variational nature is being developed based on the theory of ill-posed problems.

The construction of a rationalizing utility function is called the inverse problem of MDTh in accordance with ill-posed problems in natural sciences. But the utility function as such is an auxiliary object of the demand theory, and the main one is the demand correspondence or its regular case - the demand function. Accordingly, for verification MDTh in the case of regular demand, it is sufficient to prove the existence of the rationalizing utility function through revealing the integrability properties for the demand.

The rationalizing in the ideal case, when data (9) are exact, means the inclusion $x^{t} \in D\left(p^{t}, e_{t}\right)$, and if the correspondence $D$ is assumed to be a single-valued demand function $x(\cdot, \cdot)$, then the rationalizing means the equality $x^{t}=x\left(p^{t}, e_{t}\right)$. Accounting for the data (9) errors requires correcting the definition of their rationalizability. The correcting depends on the classes of utility and demand functions desired, as well as on the verification method and information about data errors.

\subsection{Parametric verification}

The first method for verifying the MDTh is the Parametric Demand Analysis (PDA), which short history and contemporary state are presented above, in paragraph 2.2.2. Here, the most desirable object is the total demand for all commodities of the studying market in some parametric class of continuously differentiable functions, which is denoted further by $x(p, e ; w)$, where $w=\left(w_{1}, \ldots, w_{k}\right)$ is a vector of parameters from an admissible set $W$. The assumption of collective rationality, imposed on the market demand functions $x(\cdot, \cdot ; \cdot)$ as the integrability conditions, was considered as a highly probable property (but not accepted by economic theory) in the period before Gorman's discovery of 1953 and became a heuristic after that. The undoubted advantage of PDA is the analytical form of demand systems, which allows one to reveal the marginal characteristics of market demand, such as elasticity and properties of substitution-complement between commodities. 
The works of Stone and his followers on complex analysis of market demand using LES are equivalent to the Geary collective utility function maximization. Accordingly, all the market demand analysis results using LES as the demand study hypothesis that have been validated, see (Deaton, 1975), also support the MDTh.

Subsequent development of PDA suggested more sophisticated demand system, partially noted in 2.2.2. These systems usually represent market demand functions in the budget share form and use the indirect utility function or the expenditure function (named also the 'cost function'). As a rule, they satisfy the integrability conditions, as well as the conditions of aggregation over consumers (households). They are exhaustively examined in the presented above and other literature. The integrability conditions provide a constructive verification of the MDTh by the corresponding demand systems approved in the applications, without constructing a utility function (which exists). The conditions of aggregation over consumers restrict the class of such systems, but all the works of Deaton and his followers on applied demand analysis factually approve the applicability of IDTh to market demand, at least in the cases considered, despite the over-complication of the analysis technique by these conditions. These constraints should be omitted within the framework of the MDTh.

The main shortcoming of PDA is that the unsuccessful outcomes of the given trade statistics analysis with a finite set of parameterized demand systems do not give grounds for rejecting the hypothesis of this statistics rationalizability.

\subsection{Nonparametric verification}

Revealing the question about rationalizing a given trade statistics in the general class of locally nonsatiated utility functions in principle, as well as constructive, is possible within the nonparametric demand analysis (NPDA) of Afriat-Varian (Afriat, 1967; Varian: 1982, 1983). NPDA was created within the IDTh, but it can be used as a logical tool for the market trade statistics. The NPDA experience is reflected in papers: seminal (Varian, 1982) and survey (Barnet and Serletis, 2008), among others. The survey highlights the main problem of the nonparametric approach that is the lack of methods for the data errors accounting. In this subsection, a variant of NPDA is presented, which solves the inverse problem of the MDTh with ensuring the continuity of the calculation results' dependence on data errors. This means the regularizing property of the variant.

\subsubsection{The modified Afriat's theorem and inequalities}

Hall Varian in his papers $(1982,1983)$, based on the fundamental findings of Sydney Afriat (1967), presented several criteria for rationalizing trade statistics in a very wide class of utility functions without assumptions about their quasiconcavity and differentiability.

The main NPDA facts in a modified Varian's (1982) setting that are being used in the presented here methods for verifying the MDTh and constructing Konüs indexes are as follows.

Definition 3. (Varian 1982) A utility function $u(\cdot)$ rationalizes trade statistics (9), if

$$
u\left(x^{t}\right)=\max \left\{u(x):\left\langle p^{t}, x\right\rangle \leq e_{t}, x \geq 0\right\}, \quad t=\overline{0, T} .
$$

Let's introduce the "cross-expenditures" $e_{t s}=\left\langle p^{t}, x^{s}\right\rangle$ and coefficients 


$$
a_{t s}=\left\langle p^{t}, x^{s}-x^{t}\right\rangle=e_{t s}-e_{t}
$$

Theorem 2. (Afriat's Theorem). The following conditions are equivalent:

1) there exists a locally nonsatiated utility function that rationalizes data (9);

2) there exist positive numbers $u_{t}, \lambda_{t}, t=\overline{0, T}$, such that

$$
u_{s}-u_{t}-\lambda_{t} a_{t s} \leq 0, \quad s, t=\overline{0, T} \wedge s \neq t
$$

3) the continuous, concave, monotone increasing function

$$
\bar{u}(x)=\min _{\tau}\left\{u_{\tau}+\lambda_{\tau}\left\langle p^{\tau}, x-x^{\tau}\right\rangle\right\}
$$

rationalizes the data (9).

The numbers $\left\{u_{t}, \lambda_{t}\right\}$ are called Afriat's numbers, which are the values of the rationalizing utility function (if any) and the corresponding Lagrange multiplier, i.e., $u_{t}=u\left(x^{t}\right), \lambda_{t}=\lambda\left(p^{t}, e_{t}\right)$; and function (12) is called the Afriat's function.

Remark 1. Another equivalent condition, which is Afriat's (967) 'cyclical consistency' or Varian's (1982) 'Generalized axiom of revealed preference' (GARP), used by many authors, is omitted here, because Afriat's theorem don't take into account the inevitable errors in trade statistics (9), and an effective computational procedure for such accounting when testing this condition is not known. Instead, error accounting is easily fulfilled in criterions based on Afriat's inequalities (11), as shown below.

Remark 2. The third condition is a constructive equivalent to the Afriat-Varian's condition of existence of "a nonsatiated, continuous, concave, monotonic utility function that rationalizes the data." In Varian's representations of Afriat's results the function (12) has only a technical meaning wen proving the latter condition as an example of such a function. Below, this function works effectively when constructing economic indexes and verifying the MDTh.

Remark 3. Chiappori and Rochet (1987) established a condition (Strong Axiom of Revealed Preferences) that ensure the existence of a strictly increasing, infinitely differentiable, strictly concave utility function defined on a compact subset of the commodity space containing data (9), which rationalizes the data. Analysis of their proof shows that this condition is equivalent to the positive solvability of the system of Afriat's strict inequalities $u_{s}-u_{t}-\lambda_{t} a_{t s}<0{ }^{24}$. Besides, the authors' proof is constructive and based on the integral convolution method for functions smoothing. So, if one has a utility function that rationalizes inexact data (9), and Afriat's inequalities (11) are fulfilled, then these inequalities can be relaxed by adding an arbitrarily small value to the right-hand side of (11). Such a relaxed Afriat's inequality system will have a nonempty set of interior points and provide the existence of a continuously differentiable concave and increasing utility function that rationalizes inexact data (9) on a compact subset of the commodity space containing data (9).

\footnotetext{
24 This condition can be called the "positive Slater's condition", and this equivalency is shown in (Gorbunov 2004).
} 
Thus, remarks 2 and 3 explain that the possibility of rationalizing a finite set of inexact trade data (9) in the wide class of nonsatiated and increasing utility functions can be realized in its subclass of concave and increasing utility functions (12); moreover, in the regular subclass of continuously differentiable, strictly concave, and increasing utility functions. The latter means the 'general position' of the regular subclass for real demand analysis.

\subsubsection{The homothetic Afriat's inequalities}

The homothetic preferences are important for the theory of economic indexes ${ }^{25}$ and for our methods of constructing them and verifying MDTh. Such preferences can be represented by a linearly homogeneous utility function $u(\cdot)$, and the demand functions have the structure $x(p, e)=\hat{x}(p) e$, where $\hat{x}(p)=x(p, 1)$.

Other specifics of the homothetic case: the Lagrange multiplier $\lambda$ depends only on $p$ as the superposition $\lambda(p)=u(\hat{x}(p))$, and the well-known Shephard's (1970, sec. 4.4) factorization of the cost function can be specified for the expenditure function (4) as

$$
e(p, u(x))=\frac{u(x)}{\lambda(p)}, \quad p>0, x>0
$$

Equality (13) for data $\left(p^{t}, x^{t}\right)$ takes the discrete form

$$
u_{t}=\lambda_{t} e_{t} .
$$

The corresponding Afriat's numbers $\left\{u_{t}\right\}$ and $\left\{\lambda_{t}\right\}$, satisfying (14), are called homogeneously consistent.

The use of equalities (14) in formulas (10) and (11) leads to the simplification of the latter to the homothetic Afriat's inequalities:

$$
u_{s} \leq \lambda_{t} e_{t s}, \quad s, t=\overline{0, T} \wedge s \neq t .
$$

The condition (14) allows to decompose this inequality system into two operationally equivalent 'homothetic' systems, each of them determines only one part of the Afriat's numbers, $\lambda_{t}$ or $u_{t}$ :

$$
\lambda_{s} e_{s} \leq \lambda_{t} e_{t s} ; \quad u_{s} e_{t} \leq u_{t} e_{t s} ; \quad s, t=\overline{0, T} \wedge s \neq t
$$

It suffices to consider only the first of them, which I call the $\lambda$-system. Its solution $\left\{\lambda_{t}\right\}$ determines the homogeneously consistent solution $\left\{u_{t}=\lambda_{t} e_{t}\right\}$ of the second system. The homothetic variant of Afriat's function (12) is $\min _{\tau}\left\{\lambda_{\tau}<p^{\tau}, x>\right\}$, and it is not used further.

\subsubsection{The known methods for solving Afriat's inequalities}

Afriat, after the seminal paper (1967), limited his theoretical studies of consumer demand to the case of homothetic preferences, declaring it to be the 'conceptual basis for a price index' (1972, $\mathrm{p}$. 47 ), without considering the index of quantity of consumption. He elaborated in 1970s combinatorial

\footnotetext{
${ }^{25}$ Samuelson and Swamy (1974) highlighted that homotheticity is an unrealistic property of preferences for detailed multi-commodity markets and called the homotheticity assumption as "Santa Claus hypothesis" (p. 592). On the other hand, this hypothesis is often not rejected when the verifying model (1) using aggregated and inexact data (9). This approximation can be understood as an analogue of the nonlinear processes' linearization.
} 
algorithms for solving $\lambda$-system (15) and its multiplicatively relaxed variant $\lambda_{s} e_{s} \leq \omega \lambda_{t} e_{t s}$, where the parameter $\omega \geq 0$ is the minimal of those providing the consistency of the system and called it 'critical cost efficiency' (Afriat 2014, p.43) ${ }^{26}$.

Other combinatorial algorithms for solving general (11) and $\lambda$-system (15) were elaborated by Varian $(1982,1983)$. For the $\lambda$-system, Varian adapted the known Warshall's algorithm, designed for finding the minimum cost path between the vertices of a connected graph. This method is also applied to the multiplicatively relaxed $\lambda$-system.

For general Afriat system (11), Diewert (1973) proposed the linear program (LP) - to minimize the sum of artificial variables (slack variables) additively added to inequalities (11) to ensure compatibility. Fleissig and Whitney (2005) refined this approach using only one additive relaxation variable, analogously to the relaxation-penalty method of (Gorbunov, 2001b). In the both cases of minimal relaxation, as in the combinatorial methods of Afriat and of Warshall-Varian, some solution is determined without a meaningful property. Besides, the solutions of minimally relaxed consistent systems can be unstable and, in particular, become inconsistent under small variations of their coefficients. In the mentioned and other works of other authors known to me, the problems of a reasonable choice of solutions to Afriat inequality systems and their possible instability were not considered. But the stability of numerical methods is a strict requirement.

\subsubsection{Reducing Afriat's inequalities}

The Afriat's inequality systems (11) and (15) are algebraically homogeneous. Accordingly, in the consistency case, their solution sets are unbounded cones. These sets can be essentially shortened without mispresentation of the initial inverse problem.

The general system (11) has two freedom degrees due to algebraic homogeneity and inclusion of $u$-numbers by the differences $u_{s}-u_{t}$. Due to this freedom, two conditions can be imposed on this system's solutions, and it is productive to assign

$$
\lambda_{0}=1, \quad u_{0}=e_{0} .
$$

These 'initial' conditions satisfy the homogeneous consistency condition (14), and they will also be used further for the homothetic systems (15).

Substituting conditions (16) in (11) reduces this system of homogeneous inequalities to the nonhomogeneous system with variables $\left\{u_{t}, \lambda_{t}: t=\overline{1, T}\right\}$ :

$$
\left\{\begin{array}{l}
e_{0}-\lambda_{t} a_{t 0} \leq u_{t}, \quad u_{s} \leq e_{0}+a_{0 s} \equiv e_{0 s}, \\
u_{s}-u_{t}-\lambda_{t} a_{t s} \leq 0, \quad s, t=\overline{1, T} \wedge s \neq t .
\end{array}\right.
$$

System (17) is the general reduced Afriat's inequality system. To solve the problem of rationalizing statistics (9) and constructing the rationalizing function (11), a strictly positive solution of (17) is necessary. But numerical analysis shrinks away from strict inequalities, and in what follows, the nonnegative conditions $u_{t} \geq 0, \lambda_{t} \geq 0, t=\overline{1, T}$ are imposed by default on system (17) and its variants, which will arise thereafter. If this system is nonnegatively consistent, then it has, in general,

\footnotetext{
${ }^{26}$ Afriat calls multipliers $\lambda_{t}$ "price levels" and considers an "unique logical origin" index $P_{r t}=\lambda_{r} / \lambda_{t}$ (ibid., p. vi), which is the invariant price index, presented below.
} 
a convex set in the space $R^{2 T}$ of numbers $\left\{u_{t}, \lambda_{t}: t=\overline{1, T}\right\}$, which is a polytope, possibly a singleton. This polytope is bounded with respect to $u$-numbers.

Substituting the first condition from (16), i.e., $\lambda_{0}=1$, into $\lambda$-system from (15) reduces this system of homogeneous inequalities to the nonhomogeneous reduced Afriat's inequality $\lambda$-system.

$$
e_{0} \leq \lambda_{t} e_{t 0}, \quad e_{s} \lambda_{s} \leq e_{0 s}, \quad \lambda_{s} e_{s} \leq \lambda_{t} e_{t s}, \quad s, t=\overline{1, T} \wedge s \neq t
$$

The first and the second blocks of this system determine two-side estimates for possible values of this system's solutions, and these estimates generates estimates for $u$-numbers of the solutions to (11) under the homotheticity condition (14):

$$
\frac{e_{0}}{e_{t 0}} \leq \lambda_{t} \leq \frac{e_{0 t}}{e_{t}}, \quad \frac{e_{0} e_{t}}{e_{t 0}} \leq u_{t} \leq e_{0 t}, \quad t=\overline{1, T}
$$

Estimates (19) for the nonnegatively consistent system (18) mean that system (11) with conditions $u_{t}=\lambda_{t} e_{t}$ has a convex set of solutions, which is a bounded polytope, possible a singleton, in $R^{2 T}$.

\subsubsection{Regularization of Afriat's inequalities}

As noted in Remark 1, Afriat's theorem and its reconsideration in Varian's papers $(1982,1983)$ don't account for inevitable errors in trade statistics (9). Due to these errors and/or the inadequacy of model (1) to the market under study the inequality systems (11) and (15) can be inconsistent. Besides, the solution sets of these systems, considered as multi-valued solution mapping, can be unstable concerning data variations. This instability means the absence of the compactly Hausdorff continuity ${ }^{27}$ of the solution sets from data. A multi-valued mapping locally continuous by Hausdorff is called regular. A sufficient regularity condition for the solution mappings of convex inequality systems, to which systems (11) and (15) belong, is the consistency of the corresponding systems of strict inequalities, i.e., Slater's condition. Due to the typical irregularity of the Afriat's systems, the problem of their solution should be considered ill-posed. Such problems should be regularized, i.e., to approximate by a problem with a single solution that continuously depends on the initial data, using additional information about the desired solution and, if any, errors of data.

Standard econometric methods for verifying mathematical models with inexact data are based on the assumption of the probabilistic character of their errors and, respectively, using the methods of mathematical statistics. The probabilistic approach is approved mainly for financial markets (Lee et al., 2019), but statistics of consumer markets generally do not provide probabilistic characteristics for errors $^{28}$. In this case, the methodology for ill-posed (irregular) problems, developed in natural science and engineering (Tikhonov and Arsenin, 1977; Gorbunov: 1991, 2001a), can be used.

${ }^{27}$ A multi-valued mapping $\mathrm{A}(\cdot)$ from a vector space $X$ to a space $\Lambda$ with metric $\rho(\cdot, \cdot)$ is called Hausdorff continuous at a point $x^{0}$, if $h\left(\mathrm{~A}(x), \mathrm{A}\left(x^{0}\right)\right) \rightarrow 0$ for $x \rightarrow x^{0}$, where for $A \subseteq \Lambda$ and $B \subseteq \Lambda$ the Hausdorff metric $h(A, B)=$ $\max \{\beta(A, B), \beta(B, A)\}, \beta(A, B)=\sup \{\inf \{\rho(a, b): b \in B\}: a \in A\}$.

${ }^{28}$ In view of the absence of 'precise aggregation' methods for multi-commodity trade data, provided by statistical services, the notions 'precise aggregate data' and their 'error levels' have not objective definitions. 
The regularization of the solution problem for an irregular inequality system (Gorbunov: 1999, 2001a) means, firstly, its approximation by a regular inequality system, and secondly, introducing a choice rule for the desired solution, which ensures its uniqueness and continuous dependence on the initial data. The method for regularization and solution of Afriat's systems presented further is the developing the relaxation-penalty method for degenerate inequalities and extremal problems (Gorbunov: 2001b, 2004, 2015). In recent papers (Gorbunov and Lvov 2019; Gorbunov et al., 2020), this penalty function type method has been decomposed into a two-step one. The first step is the regularization of Afriat's inequalities, and the second is the statement of an extremal problem for choosing a solution with the desired properties.

To clarify whether system (17) or (18) is regular and, with the regularity absence, to regularize it, the additive relaxation parameter $r$ is introduced in the system's right-hand side. It is also useful to provide the value $r$ independence with respect to the scale of data (9). For this, the relaxation terms in inequalities numbered $t$ or $s$ are $r e_{t}$ or $r e_{s}$, respectively, and those numbered $(s, t)$ the terms are $r \sqrt{e_{s} e_{t}}$ :

$$
\begin{cases}-u_{t}-\lambda_{t} a_{t 0} \leq-e_{0}+r e_{t}, & u_{s} \leq e_{0 s}+r e_{s}, \\ u_{s}-u_{t}-\lambda_{t} a_{t s} \leq r \sqrt{e_{s} e_{t}}, \quad s, t=\overline{1, T} \wedge s \neq t .\end{cases}
$$

Thus, general system (17) is transformed to the general relaxed normalized Afriat's system. The relaxed normalized $\lambda$-system is deduced from (20) by substituting homogeneity conditions (14):

$$
-\lambda_{t} e_{t 0} \leq-e_{0}+e_{t} r, \quad \lambda_{s} e_{s} \leq e_{0 s}+r e_{s}, \quad \lambda_{s} e_{s}-\lambda_{t} e_{t s} \leq r \sqrt{e_{s} e_{t}}, \quad s, t=\overline{1, T} \wedge s \neq t .
$$

Systems (20) and (21) are obviously consistent under sufficiently large values of the parameter $r$. Their solution sets are convex polytopes, and the set of (21) is bounded. The solution set of (20) is bounded only in $u$-numbers.

The questions about the consistency and regularity of original systems (17) and (18), as well as their regularizing, if necessary, are resolved by analysing the values of the next minimal relaxation LPs for systems (20) and (21), respectively. For system (20) the variables are $\left\{\lambda_{1}, \ldots, \lambda_{T}, u_{1}, \ldots, u_{T}, r\right\}$, and the LP is

$$
r_{\lambda u}=\arg \min \{r:(20), \lambda \geq 0, u \geq 0\}
$$

For system (21) the variables are $\left\{\lambda_{1}, \ldots, \lambda_{T}, r\right\}$, and the minimal relaxation LP is

$$
r_{\lambda}=\arg \min \{r:(21), \lambda \geq 0\} .
$$

The answers to the questions about the consistency and regularity of systems (17) and (18) depend on the values (22) and (23), respectively. Consider the general variant of system (17). If $r_{\lambda u}<0$ significantly, then system (17) is regular. In the case of $r_{\lambda u}>0$ significantly, system (17) is inconsistent and when $r_{\lambda u} \cong 0$ (is almost null), it is practically consistent, but irregular, since the corresponding system of strict inequalities can be inconsistent under small data variations ${ }^{29}$. On the

\footnotetext{
29 The fuzzy statements "a number $r$ is significantly negative/positive" mean that this negative/positive number differs from null more than a possible calculation error; and " $r$ almost equals null" means that $|r|$ does not exceed a possible calculation error.
} 
same reason, relaxed system (20) with $r=r_{\lambda u} \geq 0$ will be consistent but irregular. This system should be regularized with a modest overrelaxation $\rho>0$, which is being added to $r_{\lambda u}$ so that the total relaxation will be

$$
r=\max \left\{r_{\lambda u}+\rho, 0\right\},
$$

and the system (20) with relaxation (24) will be regular according to the theory (Gorbunov, 1999, 2001a). If $r_{\lambda u}>0$ so large that it cannot be explained by possible data errors, then the hypothesis of the adequacy of model (1) to the data (9) must be rejected. Due to the usual lack of information on data errors, the decision about the admissible level of $r_{\lambda u}$ for the acceptance of adequacy should make market statisticians.

Successful regularization of Afriat's inequalities (11) gives a constructive answer about the rationalizability of market statistics (9) by an increasing, continuous, concave utility function. Afriat's function (12), determined by a solution $\left\{u_{t}, \lambda_{t}\right\}$ of regular system (20) with parameter (24), is the approved rationalizing function of this class.

The analysis of the $\lambda$-system (18) is similar to the presented one with changing $r_{\lambda u}$ on $r_{\lambda}$, and (20) on (21). Successful regularization of $\lambda$-inequalities (15), which solutions (if any) consist only a subset of the solution set of general system (11), has fewer chances then (11); but if the relaxation value

$$
r=\max \left\{r_{\lambda}+\rho, 0\right\}
$$

is rather small and the homogeneity hypothesis can be accepted, then market statistics (9) can be rationalized by an increasing, continuous, concave, homogeneous utility function, and the problem of economic indexes and verifying the MDTh will become simpler, as shown below.

\subsubsection{Market demand indexes as addition information for the MDTh verification}

Regularized Afriat's inequalities (20) and (21) have sets of solution, and each positive solution, according to Theorem 2 or its homogeneous specification (Varian 1983), solves the inverse problem of the MDTh. However, different solutions, which are bundles of Afriat's numbers, determine essentially different utility functions which, in turn, determine different demand correspondences or functions. This non-uniqueness corresponds to the general mathematical fact of the non-uniqueness of the functions' construction from a finite set of their values, and that is revealed as underdefiniteness of functional inverse problems in their initial setting.

One of the techniques for eliminating underdefiniteness of ill-posed problems is introducing a preimage of the desired solution or some of its characteristics as an initial approximation. In the case of Afriat's inequalities, it is impossible to directly estimate any meaningful characteristics of their solutions. But these solutions, i.e., Afriat's numbers, determine Afriat's utility function (12), which determines the expenditure function (4), and this function, in turn, determines the economic price and quantity indexes presented below. Accordingly, a meaningful adjustment of the MDTh inverse problem with general statistical practice can be obtained by fitting the economic indexes, determined by the Afriat's numbers, and the best price-quantity pairs of index formulas that are Fisher indexes. This idea was partially realized in (Gorbunov, 2004) based on the relaxation-penalty method 
(Gorbunov, 2001b) using Fisher indexes as a preimage of economic indexes determined by the desired utility function.

Remind the formulas of Fisher indexes, which are determined through well-known Laspeyres and Paasche indexes:

$$
P_{s t}^{L}=\frac{e_{t s}}{e_{s}}, \quad Q_{s t}^{L}=\frac{e_{s t}}{e_{s}} \quad \text { and } \quad P_{s t}^{P}=\frac{e_{t}}{e_{s t}}, \quad Q_{s t}^{P}=\frac{e_{t}}{e_{t s}} .
$$

Here $P_{s t}^{L}$ and $Q_{s t}^{L}$ are Laspeyres indexes of prices and quantities, respectively, with the base period $s$ and the current period $t$; and the corresponding pair $\left(P_{s t}^{P}, Q_{s t}^{P}\right)$ are Paasche indexes. In most practical cases, the inequalities

$$
P_{s t}^{P} \leq P_{s t}^{L}, \quad Q_{s t}^{P} \leq Q_{s t}^{L},
$$

are being observed, and this phenomenon is called the Gerschenkron effect.

The pair of Fisher's price and quantity indexes are determined as the geometric mean of indexes (25):

$$
P_{s t}^{F}=\sqrt{P_{s t}^{L} P_{s t}^{P}}=\sqrt{\frac{e_{t s} e_{t}}{e_{s} e_{s t}}}, \quad Q_{s t}^{F}=\sqrt{Q_{s t}^{L} Q_{s t}^{P}}=\sqrt{\frac{e_{s t} e_{t}}{e_{s} e_{t s}}} .
$$

These indexes are the best in the class of formula indexes relative Fisher tests (CPIM 2004, Ch. 16).

\subsubsection{The general Konüs-Fisher indexes}

Economic (Konüs/analytical) indexes of prices and quantities are defined (Samuelson and Swamy, 1974) in general form through the expenditure function (4) as

$$
P\left(p^{t}, p^{s} ; x\right)=\frac{e\left(p^{t}, u(x)\right)}{e\left(p^{s}, u(x)\right)}, \quad Q\left(x^{t}, x^{s} ; p\right)=\frac{e\left(p, u\left(x^{t}\right)\right)}{e\left(p, u\left(x^{s}\right)\right)} .
$$

Here the vectors of quantities $x$ and prices $p$ are the corresponding reference situations. The pairs of indexes (28) with the reference situations from data (9) $\left(P_{s t}^{K L} \triangleq P\left(p^{t}, p^{s} ; x^{s}\right), Q_{s t}^{K L} \triangleq Q\left(x^{t}, x^{s} ; p^{s}\right)\right)$ and $\left(P_{s t}^{K P} \triangleq P\left(p^{t}, p^{s} ; x^{t}\right), Q_{s t}^{K P} \triangleq Q\left(x^{t}, x^{s} ; p^{t}\right)\right)$ are called Konüs-Laspeyres and Konüs-Paasche price and quantity indexes, respectively. The properties of these indexes with respect to the Fisher's tests fulfilling are presented in the paper of Samuelson and Swamy (1974), and in Diewert (1993).

In what follows, the Konüs-Fisher $(\mathrm{KF})$ indexes $P_{s t}^{K F} \triangleq\left(P_{s t}^{K L} P_{s t}^{K P}\right)^{1 / 2}, Q_{s t}^{K F} \triangleq\left(Q_{s t}^{K L} Q_{s t}^{K P}\right)^{1 / 2}$ will be used. Due to the definitions (28) and the equality $e\left(p^{s}, u\left(x^{s}\right)\right)=e_{s}$, they are:

$$
P_{s t}^{K F}=\sqrt{\frac{e\left(p^{t}, u_{s}\right) e_{t}}{e_{s} e\left(p^{s}, u_{t}\right)}}, \quad Q_{s t}^{K F}=\sqrt{\frac{e\left(p^{s}, u_{t}\right) e_{t}}{e_{s} e\left(p^{t}, u_{s}\right)}} .
$$

Thus, the problem of constructing Konüs indexes is reduced to calculating the values of the expenditure function on statistical data $e\left(p^{t}, u_{s}\right)$ and $e\left(p^{s}, u_{t}\right)$. 


\subsubsection{Calculating the expenditure function}

Problem (4) for calculating the expenditure function $e(\cdot, \cdot)$ is rather simple with Afriat's utility function (12). Really, the values $e(p, w)$ of this function are defined by convex program (4), and with function (12), the constraints in (4) are obviously represented as a system of inequalities

$$
\left\langle p^{0}, x\right\rangle \geq w, \lambda_{\tau}\left\langle p^{\tau}, x\right\rangle \geq w-u_{\tau}+\lambda_{\tau} e_{\tau}, \tau=\overline{1, T}, \quad x \geq 0 .
$$

So, calculating $e(p, w)$ is reduced to minimizing the linear function $\langle p, \cdot\rangle$ under linear conditions (30), and program (4) becomes the linear program

$$
e(p, w)=\min \{\langle p, x\rangle:(30)\} .
$$

Theorem 3. The LP(31) is solvable for any positive parameters $\left\{p^{\tau}, w, \lambda_{\tau}, u_{\tau}\right\}$.

Proof. The sufficient conditions for the LP solvability are the consistency of the corresponding constraints and the boundedness from below of the function to be minimized. The first condition is

provided by the non-negativity of the function $\langle p, \cdot\rangle$ in $R_{+}^{n}$, and the system (30) consistency follows from the global unboundedness from above of the left-hand sides of the system's inequalities.

To compute a pair of KF indexes (29), it is necessary to solve two LP (31) with parameters $\left(p^{t}, u_{s}\right)$ and $\left(p^{s}, u_{t}\right)$.

\subsubsection{Invariant indexes}

In the case of homothetic preferences, due to factorization (13) of the expenditure function, the formulas for Konüs indexes (28) take the form

$$
P\left(p^{t}, p^{s} ; x\right)=\frac{\lambda\left(p^{s}\right)}{\lambda\left(p^{t}\right)}, \quad Q\left(x^{t}, x^{s} ; p\right)=\frac{u\left(x^{t}\right)}{u\left(x^{s}\right)} .
$$

Due to the independence of these indexes from the reference situations $x$ and $y$, Samuelson and Swamy (1974) called them "invariant indexes." They are determined directly by the Afriat's numbers $\lambda_{t}=\lambda\left(p^{t}\right)$ and $u_{t}=u\left(x^{t}\right)$, and formulas (32) can be rewritten, using also homogeneity condition (14) and the renaming of indexes $P\left(p^{t}, p^{s} ; x\right) \equiv P_{s t}, \quad Q\left(x^{t}, x^{s} ; p\right) \equiv Q_{s t}$ :

$$
P_{s t}=\frac{\lambda_{s}}{\lambda_{t}}, \quad Q_{s t}=\frac{u_{t}}{u_{s}}=\frac{\lambda_{t} e_{t}}{\lambda_{s} e_{s}} .
$$

Thus, invariant price and quantity indexes $\left(P_{s t}, Q_{s t}\right)$ are determined only by $\lambda$-numbers. It is known, that they satisfy all Fisher's tests, as well as transitivity. So, invariant indexes (33) are really the ideal indexes. In addition, estimates (19) of $\lambda$-numbers in view of equality $P_{0 t}=1 / \lambda_{t}$ and definitions (25) give estimates for the invariant indexes by Laspeyres and Paasche indexes:

$$
P_{0 t}^{P} \leq P_{0 t} \leq P_{0 t}^{L}, \quad Q_{0 t}^{P} \leq Q_{0 t} \leq Q_{0 t}^{L} .
$$


It is clear that subindex 0 in (34) can be changed by arbitrary $s$, and Gerschenkron effect (26) should be fulfilled for the homothetic preferences every time.

It was noted in paragraph 4.2.2 (in the footnote) that homogeneity is an unrealistic property of preferences, but this hypothesis is often not rejected when verifying model (1) using aggregated and inexact data (9).

\subsubsection{The meaningful solving Afriat's inequalities}

As noted in paragraph 4.2.6, an adjustment of the MDTh inverse problem with statistical practice can be obtained by fitting the economic indexes determined by the Afriat's numbers to Fisher indexes (27). Other methods for meaningful solving Afriat's inequalities are based on imposing some properties on the creating indexes, e.g., "optimistic" and "pessimistic" (Gorbunov and Lvov, 2019).

Consider first the case of homothetic preferences as a simpler verification problem than the general one, and which gives an approximation for the latter. Let the value $r_{\lambda}$ of minimal relaxation LP (23) be acceptable for considering model (1) with the homogeneity hypothesis as adequate to trade statistics (9), i.e., the question about homogeneity verification is answered positively in principle, but the constructive answer on this stage is ambiguous due to the multiplicity of solutions to $\lambda$-system (21) with relaxation $r=r_{\lambda}+\rho$.

To pose the calculation problem for system (21) about the fitting of invariant indexes and Fisher ones, it is necessary to take into account that the system solution $\lambda=\left(\lambda_{1}, \ldots, \lambda_{T}\right)$ can be considered as a bundle of inverse basic invariant indexes $\left(P_{10}, \ldots, P_{T 0}\right)$ considering as a vector of $R^{T}$. Accordingly, using the Euclidean metric as a fitting measure, the quadratic problem $(\mathrm{QP})$ on the set of positive solutions to system (21) is being posed:

$$
\lambda^{F}=\arg \min \left\{\sum_{t=1}^{T}\left(\lambda_{t}-P_{t 0}^{F}\right)^{2}:(21)\right\} .
$$

Problem (35) is well-posed according to the corollary of Theorem 1 from (Gorbunov, 2001a) in view of the regularity and convexity of the admissible set of solutions to (21) and the strict convexity of the minimized function. Solution $\lambda^{F}$ can be characterized as an "objective solution", since its components are invariant indexes $\left(P_{10}, \ldots, P_{T 0}\right)$, and these indexes are nearest to the best formula indexes $\left(P_{10}^{F}, \ldots, P_{T 0}^{F}\right)$ in the common professional sense.

Invariant indexes with characteristic "optimistic" have, on assumption, the final pair $\left(P_{0 T}, Q_{0 T}\right)$ with the maximal value of price index $P_{0 T}$ and minimal value of quantity index $Q_{0 T}$. The "pessimistic indexes" have inverse properties: the maximal index $P_{0 T}$ and minimal index $Q_{0 T}$. Thus, both these problems are two-criterial in essence. However, their target functions are

$$
P_{0 T}=1 / \lambda_{T}, Q_{0 T}=\frac{\lambda_{T} e_{T}}{e_{0}},
$$


and maximizing the former and minimizing the latter are obtained simultaneously by maximizing $\lambda_{T}$. Accordingly, the correspondent problems are one-criterial LPs: the optimistic choice is ensured by maximizing $\lambda_{T}$, and the pessimistic choice is ensured by minimizing $\lambda_{T}$, both under constraints (21).

Similar problems for the general normalized Afriat's system (20) with relaxation (24) and using general Konüs-Fisher indexes (29) were suggested in (Gorbunov et al. 2020). The general case should be resolved if the homogeneity hypothesis was rejected due to an unacceptably great value $r_{\lambda}$ of the minimum relaxation LP (23), or when a more adequate investigation of the market, free from the "Santa Claus" hypothesis, is desired. Here, instead of the basic invariant indexes, basic Konüs-Fisher indexes

$$
P_{0 t}^{K F}=\sqrt{\frac{\operatorname{ex}\left(p^{t}, e_{0}\right) e_{t}}{e_{0} \operatorname{ex}\left(p^{0}, u_{t}\right)}}, \quad Q_{0 t}^{K F}=\sqrt{\frac{\operatorname{ex}\left(p^{0}, u_{t}\right) e_{t}}{e_{0} \operatorname{ex}\left(p^{t}, e_{0}\right)}}, \quad t=\overline{1, T},
$$

should be used.

Thus, the problem for system (20) about fitting Konüs-Fisher indexes (36) to Fisher ones is the non-linear program

$$
\left(\lambda^{F}, u^{F}\right)=\arg \min \left\{\sum_{t=1}^{T}\left(P_{0 t}^{K F}-P_{0 t}^{F}\right)^{2}+\left(Q_{0 t}^{K F}-Q_{0 t}^{F}\right)^{2}:(20)\right\} .
$$

Solution $\left(\lambda^{F}, u^{F}\right)$ to the problem (37) can be also characterized as an "objective solution", since its components determine Konüs-Fisher indexes (36), and these indexes are nearest to the formula Fisher indexes.

The solution of the general Afriat's system (20) with relaxation (24) providing the corresponding Konüs-Fisher indexes $\left(P_{0 T}^{K F}, Q_{0 T}^{K F}\right)$ the characteristics "optimistic" and "pessimistic", determined analogously as above, can also be resolved as a one-criterial LP. The "optimistic" pair should have the minimal price index $P_{0 T}^{K F}$ and maximal index $Q_{0 T}^{K F}$. The "pessimistic" pair have inverse properties: the maximal $P_{0 T}^{K F}$ and the minimal $Q_{0 T}^{K F}$. The simplification of these criteria, which nonlinearly depend on Afriat's number $u_{T}$, is based on the character of these dependencies. Both indexes $\left(P_{0 T}^{K F}, Q_{0 T}^{K F}\right)$ depend on the value $e x\left(p^{0}, u_{T}\right)$ of the expenditure function $e x(p, w)$, and the latter is an increasing function of the second argument. Accordingly, formulas (36) show that with increasing $u_{T}$, price index $P_{0 T}^{K F}$ increases, and quantity index $Q_{0 T}^{K F}$ decreases.

Thus, the problems of solving the general system (20), (24) that provide the characteristics "optimistic" and "pessimistic" for the corresponding Konüs-Fisher indexes $\left(P_{0 T}^{K F}, Q_{0 T}^{K F}\right)$, as in the homogeneity case, are LP ones. The former is the maximization of $u_{T}$ under constraints (20), (24), and the latter is the minimization of $u_{T}$ under the same constraints.

It is worth noting that the last two problems of solving the general Afriat's system are quite computationally complex due to the possible unboundedness of its solution set (with respect to $\lambda$ numbers). In the case when the target is determined by the final indexes $\left(P_{0 T}^{K F}, Q_{0 T}^{K F}\right)$, as in the last 
two problems, the rows of indexes $\left\{P_{0 t}^{K F}\right\}$ and $\left\{Q_{0 t}^{K F}\right\}$ can be sawtooth in nature. In this case, introducing techniques for their smoothing is required ${ }^{30}$.

So, the methods for verifying the MDTh with simultaneous constructing invariant and general Konüs (analytical) indexes with accounting their desired properties are presented briefly. A full presentation of these problems deserves a special paper in the econometrics setting.

\section{Example: Giffen demand}

Giffen demand is paradoxical consumer demand for some food product, when the demand for it increases as its price rises. The name of the paradox is connected with a Scottish statistician Robert Giffen who investigated such a kind of demand on inferior food products (bread, potato) by poor people. One example is a demand for potatoes during the great famine in Ireland 1845-47, when a massive disease of potato plantations occurred, and own potatoes was replaced on the market by more expensive imported ones. Below, Giffen demand model from (Gorbunov, 2015, p. 46-48) is represented. This demand is investigated for integrability, and the nonparametric verification of MDTh is demonstrated on the corresponding statistics.

\subsection{Model of Giffen demand}

Assume that meat and potatoes are the main food products of some part of the population, with meat being preferable to potatoes, and suppose that the nutritional value (caloric content) of a kilogram of meat and a kilogram of potatoes is 2.5 and 1 conventional food unit, respectively. The minimum requirement of a person is 10 food units. To avoid big numbers, consider a population with 1 person instead of a market SEC. The person's total expenditure for food is equal to $e$ monetary units. Prices for meat and potatoes are marked with positive vector $p=\left(p_{1}, p_{2}\right)$. It is required to determine the dependence of the purchase quantities of meat $x_{1}$ and potatoes $x_{2}$ on prices and expenditure, i.e., the demand function $x(p, e)$.

Thus, the conditions for the person's choice are inequalities

$$
2.5 x_{1}+x_{2} \geq 10, \quad p_{1} x_{1}+p_{2} x_{2} \leq e, \quad x_{i} \geq 0, i=1,2 .
$$

These conditions determine the admissible set $X(p, e) \subseteq R_{+}^{2}$, and the rational choice of the person is the point $x^{*}=x(p, e) \in X(p, e)$ with the maximal first coordinate. This choice can be refined as a solution to the LP with an objective function $f(x)=x_{1}$ and constraints (38).

Graphical analysis of the posed two-dimensional LP shows that the problem with positive parameters $(p, e)$ has a unique solution $x(p, e)$, which is the vertex of the polytope $X(p, e)$, and this vertex is determined by the system of equations

$$
2.5 x_{1}+x_{2}=10, \quad p_{1} x_{1}+p_{2} x_{2}=e .
$$

The solution to this system is the functions

\footnotetext{
${ }^{30}$ Two such techniques are introduced in (Gorbunov et al. 2020).
} 


$$
x_{1}(p, e)=\frac{e-10 p_{2}}{p_{1}-2.5 p_{2}}, \quad x_{2}(p, e)=\frac{10 p_{1}-2.5 e}{p_{1}-2.5 p_{2}} .
$$

These functions are the demand functions in the domain of their nonnegativity. This domain is the solution set of the inequality system

$$
e-10 p_{2} \geq 0,10 p_{1}-2.5 e \geq 0, p_{1}-2.5 p_{2}>0 .
$$

Consider the mathematical and economic properties of the demand functions (40). First, the dependence of demand on expenditure $e$ is important. This dependence for the first function $x_{1}(p, e)$ is an increase, and for the second $x_{2}(p, e)$, a decrease. Accordingly, meat is a valuable $e^{31}$ commodity and potatoes are an inferior one. Secondly, the dependence of the demand for a commodity on its price is also important. This dependence for meat is a decrease, and for potatoes it is an increase. Accordingly, meat is a normal commodity and potatoes are a Giffen commodity, i.e., a commodity with paradoxical, or Giffen demand.

Thus, the presented example of Giffen demand is generated by LP. The question arises, is this demand rationalizable? Above, in paragraph 3.3, the necessary and sufficient conditions for the rationalizability of a regular demand function were represented as the integrability conditions: homogeneity of degree zero, expenditure identity, negative semidefiniteness and symmetry of its Slutsky matrix. The first condition for functions (40) is obvious, the second is laid in conditions (39), and the third needs in checking.

Applying formula (7) for functions (40) gives the trivial form of the Slutsky matrix:

$$
S(p, e)=\left[\begin{array}{ll}
0 & 0 \\
0 & 0
\end{array}\right] .
$$

The null-matrix is symmetric and negative semidefinite. Accordingly, Giffen demand (40) satisfies the integrability conditions, and there is a regular neoclassical utility function that rationalizes this demand. This function can be strictly increasing, strictly quasiconcave, and twice continuously differentiable. But it cannot be strictly concave because of Theorem 1, which asserts that the diagonal entries are negative in such a case. And the question about the form of this function is open. The theoretical interest to find this form is that it would be a meaningful example of a quasiconcave utility function that is not strictly concavified.

\subsection{Nonparametric verification of Giffen demand}

Table 1 presents the conditional statistics for the Giffen demand model investigated analytically above. In these statistics, prices and expenditures are given, and the purchase quantities are calculated using formulas (40).

\footnotetext{
${ }^{31}$ It is known that a valuable commodity (demand increases with increasing expenditure) is also normal (demand decreases with increasing its price). Apparently, therefore, in the literature, normality is determined in these two ways.
} 


\begin{tabular}{|c|c|c|c|c|c|}
\hline $\mathrm{t}$ & $p_{1}^{t}$ & $p_{2}^{t}$ & $e_{t}$ & $x_{1}^{t}$ & $x_{2}^{t}$ \\
\hline 0 & 5 & 0.3 & 15 & 2.824 & 2.941 \\
\hline 1 & 5 & 0.5 & 15 & 2.667 & 3.333 \\
\hline 2 & 5 & 1 & 15 & 2.000 & 5.000 \\
\hline 3 & 5 & 1.2 & 15 & 1.500 & 6.250 \\
\hline 4 & 5 & 1.3 & 15 & 1.143 & 7.143 \\
\hline $4 / 0$ & 1 & 4.3 & 1 & 0.405 & 2.429 \\
\hline
\end{tabular}

Table 1. Trade statistics.

Table 2 presents the values of Paasche, Laspeyres (25), and Fisher indexes (27).

\begin{tabular}{|r|c|c|c|c|c|c|}
\hline$t$ & $P_{0 t}^{P}$ & $P_{0 t}^{L}$ & $P_{0 t}^{F}$ & $Q_{0 t}^{P}$ & $Q_{0 t}^{L}$ & $Q_{0 t}^{F}$ \\
\hline 0 & 1 & 1 & 1 & 1 & 1 & 1 \\
\hline 1 & 1.047 & 1.039 & 1.043 & 0.962 & 0.956 & 0.959 \\
\hline 2 & 1.304 & 1.137 & 1.218 & 0.879 & 0.767 & 0.821 \\
\hline 3 & 1.600 & 1.176 & 1.372 & 0.850 & 0.625 & 0.729 \\
\hline 4 & 1.909 & 1.196 & 1.511 & 0.836 & 0.524 & 0.662 \\
\hline
\end{tabular}

Table 2. Indexes of Paasche, Laspeyres, and Fisher.

For all times $t=\overline{1,4}$, the values of Paasche and Laspeyres price $\left(P_{0 t}^{P}, P_{0 t}^{L}\right)$ and quantity $\left(Q_{0 t}^{P}, Q_{0 t}^{L}\right)$ indexes in table 2 show the violation of Gerschenkron effect (26). Due to this effect is obligatory in the case of homothetic preferences, its violation means that the statistics of table 1 cannot be rationalized by a homogeneous utility function. This also means that the value $r_{\lambda}$ of minimal relaxation (23), which is a measure of the inconsistency of reduced Afriat's inequality $\lambda$ system (18), should be significantly positive, i.e., it cannot be explained as a consequence of possible data and/or calculation errors. Really, the LP solution (23) gives $r_{\lambda}=0.1701$, and it cannot be considered as small enough to accept the homogeneity hypothesis, especially since the values of quantities $\left(x_{1}^{t}, x_{2}^{t}\right)$ are calculated with high accuracy using formulas (40).

So, the homogeneity hypothesis for statistics of table 1 should be rejected. The general LP (22) for minimal relaxation for system (20) gives the value $r_{\lambda u}=-0.019$, which means the strict consistency of general reduced system of Afriat's inequality (17). In turn, this means the existence of a nonhomogeneous utility function that rationalizes the statistics. Such, in particular, is Afriat's function (12) determined by any positive solution to the regular system (20) with $r=0$. Due to (Chiappori and Rochet 1987) there are also strictly increasing, infinitely differentiable, strongly concave utility functions, defined on a compact subset of $R_{+}^{n}$, which rationalize data (9). These functions are uniformly close to the Afriat's function.

As noted above, some problems of solving the general Afriat's system can be quite computationally complex, and here only problem (37) of fitting Konüs-Fisher indexes (36) to Fisher ones is presented. Fitting the overrelaxation parameter $\rho$, which ensures the stability of solutions to problem (37), gave the value $\rho=0.00025$. Accordingly, formula (24) gives relaxation $r=0$. 
Table 3 presents the solution to (37), which is Afriat's numbers $\left(\lambda^{F}, u^{F}\right)$, and corresponding "objective" Konüs-Fisher indexes. As one can see, the latter coincide with Fisher formula indexes presented in table 2 . This coincidence can be explained by the shortness of the statistics. However, with its extension, KF indexes remain very close to Fisher indexes. This closeness is an additional advantage of the latter over other formula indexes.

\begin{tabular}{|r|c|c|c|c|}
\hline $\mathrm{t}$ & $\lambda^{F}$ & $u^{F}$ & $P_{0 t}^{K F}$ & $Q_{0 t}^{K F}$ \\
\hline 0 & 1 & 15.0 & 1 & 1 \\
\hline 1 & 1.138 & 14.3 & 1.043 & 0.959 \\
\hline 2 & 1.734 & 11.5 & 1.218 & 0.821 \\
\hline 3 & 2.274 & 9.3 & 1.372 & 0.729 \\
\hline 4 & 184651 & 7.7 & 1.511 & 0.662 \\
\hline
\end{tabular}

Table 3. Objective Konüs-Fisher indexes.

\section{Conclusion}

The paper has presented a way out of the reductionist impasse of the neoclassical demand theory, where it turned out to be impossible to create a market demand theory and an equilibrium theory, adequate to reality and of practical interest. The presented way out is a revision of the IDTh within the framework of the scientific holistic methodology while retaining the formal existing demand theory. This revision is in line with the desire of the founders of neoclassical Economics, Jevons and Walras, to create it on general scientific principles, and the holistic approach does not reject the basic neoclassical principles, which are optimality in the behaviour of economic agents and equilibrium in their rational interactions. Individuals can be thought as boundedly rational agents in accordance with Simon (1972), and the transfer of rationality to the SEC of the studied market is consistent with early Jevons (1866).

The meaningful economic and general scientific arguments for the revision are given, and the verification problem for the presented MDTh is considered in two variants: in parametric classes of smooth utility functions and in the general (nonparametric) class of nonsatiated utility functions. The parametric verification of the MDTh is factually, though implicitly, have fulfilled by the applied demand analysis of Stone-Deaton in a lot of positive implementations. In addition, this analysis can be freed from the problem of aggregation over consumers and thus expand its capabilities.

The presented nonparametric method for MDTh verification takes into account the typical lack of information about errors in standard trade statistics, and it is elaborated within the framework of the theory and methods for ill-posed problems. It is also a variative method for calculating the economic indexes of market demand. It allows, within the framework of the admissible arbitrariness of constructing the utility function, to adjust the indexes depending on the goals of economic analysis of various socio-political groups.

Acknowledgment: The author thanks Alexander Lvov for calculating the Example in the Wolfram Mathematica system.

Funding: This work is supported by the Russian Foundation for Basic Research [proj. № 19-01000972]. 


\section{References}

Afriat, S. N. (1967). 'The construction of utility functions from expenditure data', International Economic Review, vol. 8, pp. 67-77.

Afriat, S. N. (1972). 'The theory of international comparisons of real income and prices', Ch. 1, in: D. J. Daly (ed.), International Comparisons of Prices and Output, New York: NBER, pp. 13-84. http://www.nber.org/books/daly72-1 (Accessed 05.08.2021)

Afriat, S. N. (2014). The Index Number Problem: Construction Theorems, Oxford: Oxford Univ. Press. MR3185418

Allais, M. (1990). 'La science economigue d'aujourd'hui et les faits', Revue des Deux Mondes, June, pp. 54-74.

Anderson, P.W. (1972). 'More is different: Broken symmetry and the nature of the hierarchical structure of science', Science, vol. 177, pp. 393-395. DOI: 10.1126/science.177.4047.393

Arrow, K.J. (1994). 'Methodological individualism and social knowledge', The American Economic Review, vol. 84(2), pp. 1-9.

Arrow, K.J., Debreu, G. (1954). 'Existence of an equilibrium for a competitive economy', Econometrica, vol. 22(3), pp. 265-290. DOI:10.2307/1907353

Ashmanov, S.A. (1984). Introduction to Mathematical Economics, Moscow: Nauka (Russian).

Avriel, M., Diewert, W.E., Schaible, S. and Zang, I. (2010). Generalized Concavity. Classics in Applied Mathematics, 63. SIAM, Philadelfia [reprint ed. Plenum Press, New York, 1988]

Balasko, Y. (2016). Foundations of the Theory of General Equilibrium, 2nd edn. Hackensack, New Jersey: World Scientific. https://doi.org/10.1142/9480. Zbl 1350.91004

Barnett, W.A. and Serletis, A. (2008). 'Consumer preferences and demand systems', Journal of Econometrics, vol. 147(2), pp. 210-224. https://doi.org/10.1016/j.jeconom.2008.09.009

Belyanova, E. and Nikolaenko, S. (2016). 'Paradoxes of Theory and the Real Economy: The Nobel Prize in Economics 2015', HSE Economic Journal, vol. 20(1), pp. 175-190. https://ej.hse.ru/en/2016-20-1/179164061.html

Boumans, M. (2021). 'Retreat from normativism,' Journal of Economic Methodology, vol. 28(1), pp. 60-66. DOI: 10.1080/1350178X.2020.1868773

Boysen, O. (2019). 'When does specification or aggregation across consumers matter for economic impact analysis models? An investigation into demand systems', Empirical Economics, vol. 56, pp. 137-172. doi: 10.1007/s00181-017-1353-z

Brown, A. and Deaton, A. (1972). 'Surveys in Applied Economics: Models of Consumer Behaviour', The Economic Journal, vol. 82(328), pp. 1145-1236. doi:10.2307/2231303

Cassel, G. (1967). The Theory of Social Economy, Ney York: Augustus M. Kelley, (1 ${ }^{\text {st }}$ edn. 1918, German).

Chiappori, P.-A. and Ekeland, I. (2009). 'The microeconomics of efficient group behavior: Identification', Econometrica, vol. 77(3), pp. 763-799. DOI: 10.3982/ECTA5929

Chiappori, P.-A. and Rochet, J.-C. (1987). 'Revealed preferences and differentiable demand', Econometrica vol. 55(3), pp. 687-691. DOI: 10.3982/ECTA5929

Cherchye, L., De Rock, B. and Vermeulen, F. (2010). 'Collective household consumption behavior: Revealed preference analysis', Foundations and Trends in Economics, vol.4(4), pp. 225-312.Zbl 1248.91034

CPIM (2004). Consumer Price Index Manual: Theory and Practice. Geneva: International Labour Office. 
Deaton, A. (1975). Models and Projections of Demand in Post-War Britain. Cambridge: SpringerScience+Business Media, B.V. DOI 10.1007/978-1-4899-3113-9

Deaton, A. (1986). 'Demand Analysis', in: Z. Griliches, M.D. Intriligator (eds.), Handbook of Econometrics, vol. 3., Ch. 30, pp. 1767-1839, Amsterdam: New-Holland.

Deaton, A. and Muellbauer, J. (1980a). 'An almost ideal demand system', The American Economic Review, vol. 70, pp. 312-326

Deaton A. and Muellbauer, J. (1980b). Economics and Consumer Behavior. Cambridge and New York: University Press Cambridge.

Debreu, G. (1959). The Theory of Value: An Axiomatic Analysis of Economic Equilibrium. New Haven and London: Yale Univ. Press.

Diewert, W.E. (1993). 'The economic theory of index numbers: A survey', in: W.E. Diewert, A.O. Nakamura (eds), Essays in Index Number Theory, vol. I, pp. 177-228, Amsterdam: Elsevier.

Diewert, W.E. (2001). 'The consumer price index and index number purpose', Journal of Economic and Social Measurement vol. 27, pp. 167-248.

Dorfman, R., Samuelson, P. and Solow, R. (1958). Linear Programming and Economic Analysis, New York: McGraw-Hill.

Fleissig, A.R. and Whitney, G.A. (2005). 'Testing for the significance of violations of Afriat's inequalities', Journal of Business and Economic Statistics vol. 23, pp. 355-362. DOI 10.1198/073500104000000253

Friedrichsen, J. König, T. and Tobias L. (2021). 'Social status concerns and the political economy of publicly provided private goods', The Economic Journal, vol. 131(633), pp. 220246, https://doi.org/10.1093/ej/ueaa076.

Frisch, R. (1992). 'Statics and dynamics in economic theory', Structural Change and Economic Dynamics, vol. 3, pp. 391-401 (Norwegian orig. 1929).

Frisch, R. (1936). 'Annual survey of general economic theory: The problem of index numbers', Econometrica, vol. 4, pp. 1-38.

Geary, R. C. (1951). 'A note on "a constant-utility index of the cost of living"', The Review of Economic Studies, vol. 18(1), pp. 65-66.

Gorbunov, V.K. (1991). 'Regularization of extremum problems', USSR Computational. Mathematics and Mathematical Physics. vol. 31(2), 42-52. Zbl 0774.65037.

Gorbunov, V.K. (1999). 'Regularization of compactly solvable problems', Moscow University Computational Mathematics and Cybernetics, no 1, pp. 13-19. Zbl 1050.65504

Gorbunov, V.K. (2001a). 'Regularization of degenerated equations and inequalities under explicit data parameterization', Journal of Inverse and Ill-Posed Problems, vol. 9(6), pp. 575-594.

Gorbunov, V.K. (2001b). 'The relaxation-penalty method and degenerate extremal problems', Doklady Mathematics, vol. 62(2), pp. 219-223. Zbl 1090.90541

Gorbunov, V.K. (2004). Mathematical model of the consumers' demand: Theory and applied potential, Moscow: Economizdat. (Russian)

Gorbunov, V.K. (2013). 'To the theory of market demand: Regularity and economic equilibrium', Ekonomicheskaya Nauka Sovremennoy Rossii, vol. 63, pp. 19-36. (Russian). Zbl 1310.91089

Gorbunov, V.K. (2015). Consumers' Demand: Analytical Theory and Applications, Ulyanovsk: Publishing House of Ulyanovsk State University. (Russian).

http://www.rfbr.ru/rffi/ru/books/o_1945611 (Accessed 05.08.2021) 
Gorbunov, V.K. (2018). 'Holistic theory of economic equilibrium: modified Cassel-Wald model', Doklady Mathematics, vol. 98, pp. 537-539. DOI: 10.1134/S1064562418060121. Zbl 1419.91448

Gorbunov, V.K. (2021). 'The holistic theory of the consumer market demand', European Proceedings of Social and Behavioural Sciences, vol. 105-CDSES 2020, pp. 476-485. DOI: 10.15405/epsbs.2021.04.52

https://www.europeanproceedings.com/files/data/article/10060/13380/article_10060_13380_pd f_100.pdf

Gorbunov, V.K., Lvov, A.G. (2019). 'Inverse problem of the market demand theory and analytical indices of demand', Zhurnal SVMO, vol. 21, pp. 89-110. (Russian). DOI: 10.15507/20796900.21.201901.89-110

Gorbunov, V.K., Kozlova, L.A., Lvov, A.G. (2020). 'The construction of analytical indexes of market demand: A variative approach', Voprosy Statistiki, vol. 27, pp. 65-80. (Russian).

DOI: $10.34023 / 2313-6383-2020-27-3-65-80$

Gorman, W.M. (1953). 'Community preference fields', Econometrica, vol. 21, pp. 63-80.

Harstad, R. M., Selten, R. (2013). 'Bounded-rationality models; Tasks to become intellectually competitive', Journal of Economic Literature, vol. 51, pp. 496-511.

Hicks, J.R. (1975). Value and Capital: An Inquiry into Some Fundamental Principles of Economic Theory, New York: Oxford University Press, 2 edn (1st edn 1939).

Hicks, J. R. (1956). A Revision of Demand Theory, New York: Oxford University Press.

Hicks, J. R., Allen, R. G. D. (1934). A reconsideration of the theory of value. Economica, New Series. 1: Part I, pp. 52-76; Part II, pp. 196-219.

Hildenbrand, W. (1994). Market Demand: Theory and Empirical Evidence, Princeton: Princeton University Press. [Adobe Digital Editions version]. https://books.google.ru/books?id=sPOQjwEACAAJ\&hl=ru\&source=gbs_book_other_versions

Hillinger, C. (2008). 'Science and ideology in economic, political and social thought', Economics: The Open-Assessment E-Journal, vol. 2. http://www.economicsejournal.org/economics/journalarticles/2008-2/

Intriligator, M. (2002). Mathematical optimization and economic theory. Classics in Applied Mathematics, 39. Philadelfia: SIAM, [reprint ed. Prentice-Hall, Ney York, 1971]

http://www.ru.ac.bd/stat/wpcontent/uploads/sites/25/2019/03/404_05_Intrilligator_Mathematical-optimization-Economictheory.pdf

Jehle, G. A. and Reny, Ph. J. (2011). Advanced Microeconomic Theory. 3 edn. Harlow: Pearson.

Jevons, W. S. (1866). 'Brief account of a general mathematical theory of political economy', Journal of the Royal Statistical Society. London, XXIX, pp. 282-287.

https://socialsciences.mcmaster.ca/econ/ugcm/3113/jevons/mathem.txt

Jevons, W. S. (1957). The Theory of Political Economy, 5 edn. Ney York: Augustus M. Kelley, (1st edn 1871).

Kirman, A. (2006). 'Demand theory and general equilibrium: From explanation to introspection, a journey down the wrong road', History of Political Economy, vol. 38, pp. 246-280. https://doi.org/10.1215/00182702-2005-025

Kirman, A. (2010). 'The economic crisis is a crisis for economic theory', CESifo Economic Studies, vol. 56, pp. 498-535. 
Klein, L. R. and Rubin, H. (1947 - 1948). 'A constant-utility index of the cost of living', The Review of Economic Studies vol. 15, pp. 84-87.

Konüs, A.A. (1939). 'The problem of the true index of the cost of living', Econometrica vol. 7, pp. 1029 (Russian orig. 1924).

Lee, Ch-F., Chen, H-Y. and Lee, J. (2019). Financial Econometrics, Mathematics, and Statistics. Theory, Method, and Application, New York: Springer. Zbl 07045603

Mas-Colell, A. (1985). The Theory of General Economic Equilibrium: A Differentiable Approach, Cambridge: Cambridge Univ. Press.

Mas-Colell, A., Whinstone, M. and Green, J. (1995). Microeconomic Theory, Ney York: OUP.

Morishima, M. (1964). Equilibrium, Stability, and Growth, Oxford: Clarendon Press.

Polterovich, V.M. (1998). 'Krizis ekonomicheskoy teorii [Crisis of economic theory]', Ekonomicheskaya Nauka Sovremennoy Rossii, No. 1, pp. 46-66 (Russian).

Samuelson, P.A. (1938). 'A Note on the pure theory of consumer's behaviour', Economica, New Series, vol. 5(17), pp. 61-71.

Samuelson, P.A. (1956). 'Social indifference curves', The Quarterly Journal of Economics, vol. 70(1), pp. 1-22.

Samuelson, P.A. (1974). 'Complementarity. An essay on the 40th anniversary of the Hicks-Allen revolution in demand theory', Journal of Economic Literature vol. 12(4), pp. 1255-89.

Samuelson, P.A., Swamy, S. (1974). 'Invariant economic index numbers and canonical duality: Survey and Synthesis', The American Economic Review vol. 64, pp. 566-593.

Simon, H. (1972). 'Theories of bounded rationality', in: Decision and Organization, C. B. McGuire, R. Radner (eds.), pp. 161-176, Amsterdam: North-Holland.

Slutsky, E. (1915). 'Sulla teoria del bilancio del consumatore', Giornale degli Economisti, vol. 51, pp. 1-26. [Engl. Transl. 2012: On the theory of the budget of the consumer. Giornale degli Economisti e Annali di Economia. 71(Anno 125)(2/3), Nuova Serie, 173-200].

https://www.jstor.org/stable/pdf/43828058.pdf?seq=1]

Shephard, R.W. (1970). Cost and Production Functions, New Jersey: Princeton University Pres (1st edn 1953).

Schumpeter, J.A. (1980). Methodological Individualism. (Engl. transl. of German orig. of 1908). https://mises.org/library/methodological-individualism

Stone, R. (1945). 'The Analysis of Market Demand', Journal of Royal Statistical Society, vol. 108(3/4), pp. 286-391. DOI:10.2307/2981291.

Stone, R. (1954). 'Linear expenditure systems and demand analysis: an application to the pattern of British demand', Economic Journal vol. 64, pp. 511-527.

Tikhonov, A.N., Arsenin, V.Ya. (1977). 'Solution of Ill-Posed Problems', New York: Wiley (Russian orig. 1974).

Varian, H. (1982). 'The nonparametric approach to demand analysis', Econometrica, vol. 50(4), pp. $945-973$.

Varian, H. (1983). 'Non-parametric tests of consumer behaviour', The Review of Economic Studies, vol. 50(1), pp. 99-110.

Wald, A. (1951). 'On some systems of equations of mathematical economics', Econometrica, vol. 19, pp. 368-403 (German orig. 1936).

Walras, L. (1954). Elements of Pure Economics, London: Allen and Unwin, (1 ${ }^{\text {st }}$ French edn. Les éléments d'économie politique pure, Paris: Economica. 1877).

Zadeh, L. A. (1965). 'Fuzzy sets', Information and Control, vol. 8, pp. 338-353. 